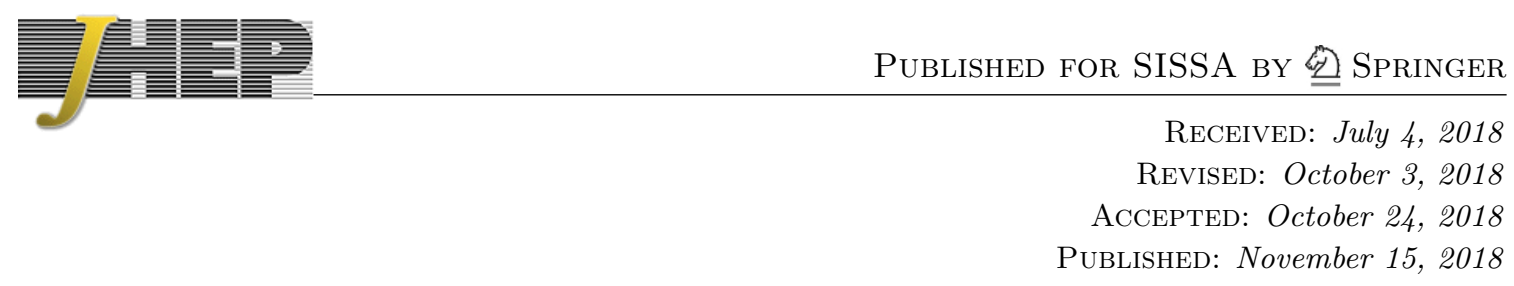

\title{
Positivity of hexagon perturbation theory
}

\author{
Burkhard Eden, ${ }^{a}$ Yunfeng Jiang, ${ }^{b}$ Marius de Leeuw, ${ }^{c}$ Tim Meier, ${ }^{a}$ Dennis le Plat $^{a}$ and \\ Alessandro Sfondrini ${ }^{b}$ \\ ${ }^{a}$ Institut für Mathematik und Physik, Humboldt-Universität zu Berlin, \\ Zum großen Windkanal 6, 12489 Berlin, Germany \\ ${ }^{b}$ Institut für theoretische Physik, ETH Zürich, \\ Wolfgang-Pauli-Straße 27, 8093 Zürich, Switzerland \\ ${ }^{c}$ School of Mathematics, Trinity College Dublin, \\ Dublin, Ireland \\ E-mail: eden@math.hu-berlin.de, jiangyu@phys.ethz.ch, \\ mdeleeuw@maths.tcd.ie, tmeier@physik.hu-berlin.de, \\ diplat@physik.hu-berlin.de, sfondria@itp.phys.ethz.ch
}

ABSTRACT: The hexagon-form-factor program was proposed as a way to compute threeand higher-point correlation functions in $\mathcal{N}=4$ super-symmetric Yang-Mills theory and in the dual $\mathrm{AdS}_{5} \times \mathrm{S}^{5}$ superstring theory, by exploiting the integrability of the theory in the 't Hooft limit. This approach is reminiscent of the asymptotic Bethe ansatz in that it applies to a large-volume expansion. Finite-volume corrections can be incorporated through Lüscher-like formulae, though the systematics of this expansion is largely unexplored so far. Strikingly, finite-volume corrections may feature negative powers of the 't Hooft coupling $g$ in the small- $g$ expansion, potentially leading to a breakdown of the formalism. In this work we show that the finite-volume perturbation theory for the hexagon is positive and thereby compatible with the weak-coupling expansion for arbitrary $n$-point functions.

KEywords: Integrable Field Theories, Supersymmetric Gauge Theory, AdS-CFT Correspondence, 1/N Expansion

ArXiv EPrint: 1806.06051 


\section{Contents}

1 Introduction 1

2 Review of hexagon form factors $\quad 3$

2.1 Form factor for a single hexagon 3

2.2 Weak-coupling expansions 4

3 Positivity of hexagon perturbation theory $\quad 7$

3.1 Naïve estimate of 't Hooft-coupling order 7

3.2 Triple scattering processes 8

$\begin{array}{lll}3.3 & \text { Improved estimate of 't Hooft-coupling order } & 10\end{array}$

3.4 Physical magnons 11

4 Application to planar BPS four-point functions 13

$\begin{array}{lll}5 & \text { Conclusions and outlook } & 17\end{array}$

$\begin{array}{lr}\text { A Algorithm for triple mirror scattering } & 19\end{array}$

$\begin{array}{ll}\text { A.1 Universal move } & 20\end{array}$

A.2 The case $S>N_{1}$ and $N_{3} \geq N_{1}$

A.3 The case $S \leq N_{1}$ and $N_{3}>N_{1} \quad 22$

A.4 The case $N_{3}=N_{1}$ and $S \leq N_{3}$

B General configurations involving physical magnons 25

\section{Introduction}

Computing correlation functions is one of the central problems in quantum field theory. For a generic interacting theory, it is impossible to calculate such observables nonperturbatively. Usually one needs to perform an expansion in certain parameters. A typical example is the small-coupling expansion around a free theory, which can be performed by well-established techniques such as Feynman diagrams. In some special theories, more powerful alternative techniques may exist which are usually based on symmetries. In integrable quantum field theories, the form-factor bootstrap approach is such an alternative. While most integrable models appear in one or two dimensions, integrability sometimes manifests itself in higher-dimensional theories too, for instance through dualities such as AdS/CFT [1-3]. One of the most prominent examples is the $\mathcal{N}=4$ supersymmetric YangMills theory (SYM), dual to type-IIB superstrings on $\mathrm{AdS}_{5} \times \mathrm{S}^{5}$. In the planar limit [4], the integrability of the spectral problem for the two-dimensional theory on the string worldsheet carries over to $\mathcal{N}=4 \mathrm{SYM}$ in the guise of an integrable spin chain [5], see e.g. 
refs. $[6,7]$ for reviews. Interestingly, it was recently realised that integrability might play a role for more general observables too. Indeed, a generalisation of the form factor approach to $\mathcal{N}=4 \mathrm{SYM}$ has been put forward in ref. [8] in terms of hexagonal tessellations; in the following we refer to this construction as the hexagon approach. This was initially proposed as a way to compute planar three-point functions involving non-protected operators. Soon after, it was realised that those techniques could be adapted to higher-point planar correlation functions $[9,10]$ and at least to some extent to non-planar observables $[11,12] .^{1}$

The hexagon approach is reminiscent of the "asymptotic" Bethe ansatz for the $\mathcal{N}=4$ SYM spin chain [16], or of the Bethe-Yang equations in two dimensional integrable QFTs: it is only exact up to exponentially-small corrections in the volume of the theory - the $R$-charge of the $\mathcal{N}=4 \mathrm{SYM}$ operators under consideration. In the spectral problem, such finite-size corrections can be interpreted as due to virtual ("mirror") particles wrapping the worlsdheet [17], similar to the Lüscher effects of relativistic theories [18, 19]. In the hexagon program, they can similarly be described as mirror particles probing the finite-size structure of the hexagon tessellation. For three-point functions, the first few finite-volume corrections can be explicitly computed and matched with small-coupling perturbation theory $[8,20$ 22 ]. Furthermore, in that context only a finite number of finite-volume corrections needs to be computed at each given order of the 't Hooft coupling expansion [21]. The situation is less clear for higher-point functions. The results of the hexagon approach for four- $[9,10]$ and five-point [23] functions and for certain non-planar observables [11, 12] match with the lowest orders of perturbation theory; such integrability computations account for the first few finite-volume corrections. Still, it was not shown so far that more complicated finite-volume effects (involving more mirror particles) can indeed be neglected at those orders. More generally, we do not know which finite-volume effects (i.e. how many mirror particles) we need to take into account at a given order of the 't Hooft-coupling expansion. Establishing this relation for general correlation functions is the aim of this paper.

The reason why this is a subtle problem - compared for instance to Lüscher corrections in the spectral problem - is that in the hexagon formalism mirror particle come in three distinct families. Indeed three of the six edges of the hexagon correspond to physical excitations, and the remaining three correspond to mirror (or anti-mirror) ones, leading to distinct kinematic regions. In the hexagon form factor, while physical processes yield non-negative powers of the 't Hooft coupling $g^{2}$ in the $g \ll 1$ expansion, mirror-anti-mirror processes yield a factor of $g^{-2}$. Naïvely, populating a hexagon with sufficiently many mirror magnons can make a process which is extremely suppressed in "large-volume" appear even at tree level $g^{0}$. Even worse, in principle we might expect contributions to processes with arbitrarily negative powers of $g^{2}$ !

Clearly such a situation would be disastrous for the hexagon program. Since several explicit computations match field theory results [9-12, 23], there should be a way to resolve this apparent issue. One possibility is that such multi-mirror-magnon processes indeed appear, but somehow cancel at appropriate orders in $g^{2}$ for correlation functions of physical

\footnotetext{
${ }^{1}$ Non-planar observables in $\mathcal{N}=4 \mathrm{SYM}$ are also being actively investigated by other integrability-based approaches, see e.g. refs. [13, 14], as well as ref. [15] for a review of earlier developments.
} 
states; this would make the formalism consistent in principle, but it would also make it almost impossible to extract physical data from the hexagon construction for generic observables without knowing a priori which processes cancel and which do not. The other much more appealing possibility is that there exist a refined estimate where, as we add more and more mirror magnons, we obtain higher and higher terms in the $g^{2}$ expansion. In this paper we show that this is the case for arbitrary (planar and non-planar) correlation functions of protected operators, obtaining an explicit formula relating the number of mirror magnons to the order in the small- $g$ expansion. We call this property the positivity of the hexagon perturbation theory. This also extends to the case of non-protected operators, which however depends more subtly on which particular correlator we consider.

The paper is structured as follows: we start by reviewing some essential features of the hexagon approach in section 2 . In section 3 we present our main result: an improved estimate for the contribution of mirror processes to the $g$-expansion, which is bounded from below, valid for any correlator of BPS operators; we also comment on the extension to nonBPS operators. In section 4 we apply these ideas to the computation of planar four-point functions of BPS operators. We conclude in section 5. We also present an alternative (algorithmic) derivation of our improved bound in appendix A.

\section{Review of hexagon form factors}

When computing four-point and higher correlation functions we need to triangulate a punctured Riemann surface (the sphere, for planar correlators). The edges of such triangles are the "mirror" edges of the hexagons. By blowing up the punctures to small circles we can add three more "physical" edges corresponding to arcs on those circles. Since we are interested in obtaining an estimate valid for any correlation functions, below we shall not make any assumption on how hexagons are glued together. Instead, we will consider a single hexagon.

\subsection{Form factor for a single hexagon}

As we mentioned, the key idea of the hexagon approach to correlation functions is to decompose any correlation function into a hexagonal tessellation. To "glue" together such a tessellation it is necessary to insert a complete basis of states for each of the three "mirror" edges. This amounts to populating the hexagon with mirror magnons. Notice that, precisely because mirror magnons arise from "gluing", they are shared by two contiguous hexagons. Taking this into account, the hexagon form factor with $\mathbf{u}, \mathbf{v}, \mathbf{w}$ sets of mirror magnons and $\mathbf{x}, \mathbf{y}, \mathbf{z}$ sets of physical magnons takes the form

$$
\text { hexagon } \sim \sqrt{\mu_{\mathbf{u}} \mu_{\mathbf{v}} \mu_{\mathbf{w}}} e^{\frac{i}{2} \tilde{E}_{\mathbf{u}} \ell_{\mathbf{u}}+\ldots} e^{i \psi \mathbf{J}+\ldots}\left\langle\mathfrak{h} \mid \mathbf{z}^{4 \gamma}, \mathbf{u}^{3 \gamma}, \mathbf{y}^{2 \gamma}, \mathbf{v}^{\gamma}, \mathbf{x}^{0 \gamma}, \mathbf{w}^{-\gamma}\right\rangle .
$$

Some comments are in order. Here $\mu$ is the mirror measure corresponding to each mirror magnon; we only assign "half" of such a measure to each hexagon, as mirror magnons are shared across two hexagons. For the same reason, we split in half the "bridge length" $\ell$ along which each magnon propagates. Next, we allow for an arbitrary chemical potential $\psi$ (for any possible magnon charge, which we collectively denote by $\mathbf{J}$ ) which is needed to 
describe four- and higher-point functions [9, 10]. The powers of $\gamma$ identify how many times we perform the mirror transformation in the notation of ref. [21]. Notice that physical particles sit at even- $\gamma$ edges, and mirror ones at odd- $\gamma$ ones (cf. figure 1). Finally, ${ }^{2}$ the hexagon form factor $\mathfrak{h}$ can then be related to the centrally extended $\mathfrak{s u}(2 \mid 2)$ (bound state) $S$-matrix $S_{i j}[24,25]$

$$
\left\langle\mathfrak{h} \mid \mathbf{z}^{4 \gamma}, \mathbf{u}^{3 \gamma}, \mathbf{y}^{2 \gamma}, \mathbf{v}^{\gamma}, \mathbf{x}^{0 \gamma}, \mathbf{w}^{-\gamma}\right\rangle \sim \prod_{i<j} h_{i j} S_{i j}
$$

up to a suitable projection in flavour space on the right-hand side [8]. The hexagon factor $h_{i j}$ for two bound states with bound state numbers $Q_{1}, Q_{2}$ is given by

$$
\begin{aligned}
h_{12} & =\prod_{k=-\frac{Q_{1}-1}{2}}^{\frac{Q_{1}-1}{2}} \prod_{l=-\frac{Q_{2}-1}{2}}^{\frac{Q_{2}-1}{2}} h\left(u_{1}^{[2 k]}, u_{2}^{[2 l]}\right), \\
h\left(u_{1}, u_{2}\right) & =\frac{x_{1}^{-}-x_{2}^{-}}{x_{1}^{-}-x_{2}^{+}} \frac{1-\frac{1}{x_{1}^{-} x_{2}^{+}}}{1-\frac{1}{x_{1}^{+} x_{2}^{+}}} \frac{1}{\sigma\left(u_{1}, u_{2}\right)} .
\end{aligned}
$$

The dressing factor $\sigma_{12}$ is given by the BES phase [26]. The purpose of this paper is to explore whether a perturbative weak-coupling expansion is compatible with the hexagon approach. In order to address this question we need to understand the weak-coupling expansion of

$$
\mathcal{S}_{i j}=h_{i j} S_{i j}
$$

i.e. of the $s u(2 \mid 2) S$-matrix dressed by the hexagon scalar factor, in different kinematic channels.

\subsection{Weak-coupling expansions}

In order to see how different pieces of eq. (2.1) scale when $g \ll 1$, let us collect here the weak-coupling expansion of the relevant quantities, and fix our conventions.

Conventions. We need to compute the $S$-matrix between magnons with different mirror orientations. For consistency, we will work with the string-frame $S$-matrix [27], see also ref. [6] for a review. We follow the conventions of [8, 23], where the bound-state $S$-matrix is normalized such that the scattering of the highest-weight fermionic state is set to unity. Moreover, we note that the dressing factor $\sigma$ for two bound states with bound-state numbers

\footnotetext{
${ }^{2}$ In order to compute a complete correlation function it would be necessary to consider all hexagons in the tessellation, sum over partitions for physical excitations, sum over all possible mirror particles and integrate over their rapidities - and normalise the result appropriately. The detail of this, as we mentioned, depend on the particular correlator we are computing; here we will not perform this procedure, as we aim at an estimate as general as possible.
} 


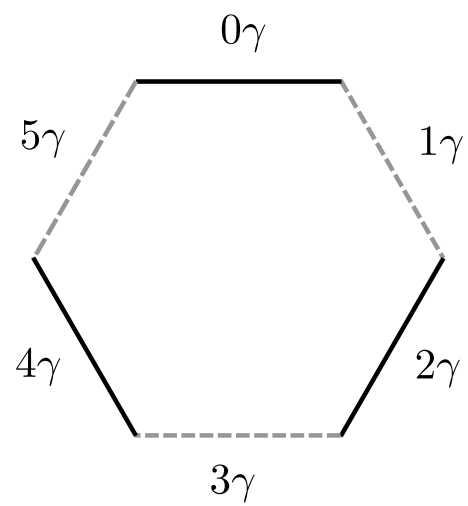

Figure 1. Three of the edges of each hexagon in a tessellation correspond to physical string states, and are denoted by solid lines. The remaining three edges correspond the "mirror channels"; physical and mirror excitations are related to each other by analytic continuation. Following ref. [8] we denote by $\gamma$ the different sheets in the analytic continuation, so that physical particles have even $\gamma$ 's and mirror ones have odd $\gamma$ 's.

$Q_{1,2}$ satisfies the following crossing equations [28]

$$
\begin{gathered}
\sigma_{12}\left(u_{1}^{2^{\gamma}}, u_{2}\right)=\left(\frac{x_{2}^{-}}{x_{2}^{+}}\right)^{Q_{1}} \frac{x_{1}^{-}-x_{2}^{+}}{x_{1}^{-}-x_{2}^{-}} \frac{1-\frac{1}{x_{1}^{+} x_{2}^{+}}}{1-\frac{1}{x_{1}^{+} x_{2}^{-}}} \prod_{k=1}^{Q_{1}-1} \frac{u_{1}-u_{2}-i \frac{Q_{2}-Q_{1}+2 k}{g}}{u_{1}-u_{2}+i \frac{Q_{2}-Q_{1}+2 k}{g}} \frac{1}{\sigma_{12}\left(u_{1}, u_{2}\right)}, \\
\sigma_{12}\left(u_{1}, u_{2}^{-2^{\gamma}}\right)=\left(\frac{x_{1}^{+}}{x_{1}^{-}}\right)^{Q_{2}} \frac{x_{1}^{-}-x_{2}^{+}}{x_{1}^{-}-x_{2}^{-}} \frac{1-\frac{1}{x_{1}^{+} x_{2}^{+}}}{1-\frac{1}{x_{1}^{+} x_{2}^{-}}} \prod_{k=1}^{Q_{1}-1} \frac{u_{1}-u_{2}-i \frac{Q_{2}-Q_{1}+2 k}{g}}{u_{1}-u_{2}+i \frac{Q_{2}-Q_{1}+2 k}{g}} \frac{1}{\sigma_{12}\left(u_{1}, u_{2}\right)} .
\end{gathered}
$$

The dressing factor respects unitarity so that $\sigma_{12}=1 / \sigma_{21}$. By using the crossing equations and unitarity we can, for instance, relate all the dressing phases between mirror and antimirror particles to $\sigma\left(u^{\gamma}, v^{\gamma}\right)$.

Expansions. Let us spell out the expansions of the different scattering matrices $\mathcal{S}$ for small coupling $g$. These can be straightforwardly checked for the scattering of fundamental magnons and, by the fusion procedure, the results carry over to bound-state $S$-matrices. ${ }^{3}$ For virtual particles with the same mirror orientation, we find that the expansion starts at order $g^{2}$

$$
\mathcal{S}\left(u_{i}^{3 \gamma}, u_{j}^{3 \gamma}\right) \sim \mathcal{S}\left(u_{i}^{\gamma}, u_{j}^{\gamma}\right) \sim \mathcal{S}\left(u_{i}^{-\gamma}, u_{j}^{-\gamma}\right) \sim g^{2} .
$$

For physical particles, we find that the expansion always starts at order 1

$$
\begin{aligned}
\mathcal{S}\left(u_{i}, u_{j}\right) & \sim \mathcal{S}\left(u_{i}^{2 \gamma}, u_{j}^{2 \gamma}\right) \sim \mathcal{S}\left(u_{i}^{4 \gamma}, u_{j}^{4 \gamma}\right) \sim g^{0} \\
\mathcal{S}\left(u_{i}^{2 \gamma}, u_{j}\right) & \sim \mathcal{S}\left(u_{i}^{4 \gamma}, u_{j}\right) \sim \mathcal{S}\left(u_{i}^{4 \gamma}, u_{j}^{2 \gamma}\right) \sim g^{0} .
\end{aligned}
$$

Next we expand the $S$-matrix for the scattering processes that involve virtual magnons from different edges

$$
\mathcal{S}\left(u_{i}^{3 \gamma}, v_{j}^{\gamma}\right) \sim \mathcal{S}\left(u_{i}^{3 \gamma}, u_{j}^{-\gamma}\right) \sim \mathcal{S}\left(u_{i}^{\gamma}, u_{j}^{-\gamma}\right) \sim g^{-2} .
$$

\footnotetext{
${ }^{3}$ We have explicitly checked all these expansions for the $Q=2$ bound-state $S$-matrix.
} 
This is the contribution that makes it possible, at least in a naïve estimate, to obtain arbitrarily negative powers of $g^{2}$ when adding mirror magnons. Finally, let us also consider the scattering between a virtual and a physical magnon. The dressed $S$-matrix is of order 1 unless the virtual magnon is on the edges that are opposite to the physical magnon

$$
\mathcal{S}\left(u_{i}^{n \gamma}, u_{j}^{(n+3) \gamma}\right) \sim \begin{cases}g^{-1} & \text { if magnon } 1 \text { is a boson } \phi^{a}, \\ g^{0} & \text { if magnon } 1 \text { is a fermion } \psi^{\alpha}\end{cases}
$$

where $n=-2,0,2 .{ }^{4}$ In the last two expansions we encounter yet another potential problem, as the negative powers of the coupling constant $g$ can potentially lead to arbitrary negative powers of the coupling constant in the hexagon expansion.

Multiple scattering. A remarkable observation which will be crucial in what follows is that the weak-coupling expansion of the scattering process between three virtual magnons may scale "better than naïvely expected". Specifically, let us take three mirror magnons from three different edges. We then find

$$
\mathcal{S}_{12}\left(u^{3 \gamma}, v^{+\gamma}\right) \mathcal{S}_{13}\left(u^{3 \gamma}, w^{-\gamma}\right) \mathcal{S}_{23}\left(v^{+\gamma}, w^{-\gamma}\right) \sim g^{-2} .
$$

We would expect this process to scale like $g^{-6}$, but it turns out that the most divergent contributions cancel out, so that the process scales four orders higher in the $g$ expansion than we would expect! This is a rather striking and unique property of the $S$-matrix: we have verified that, up to scattering six magnons, there are no further identities of this type in any mirror channel. Finally, it is also interesting to note that a similar (weaker) triple-scattering property arises also in the case where one of the magnons is physical, one virtual magnon is adjacent and the other virtual magnon is across the physical magnon,

$$
\begin{aligned}
\mathcal{S}_{12}\left(u^{3 \gamma}, v^{+\gamma}\right) \mathcal{S}_{13}\left(u^{3 \gamma}, x^{0 \gamma}\right) \mathcal{S}_{23}\left(v^{+\gamma}, x^{0 \gamma}\right) \sim g^{-2}, \\
\mathcal{S}_{12}\left(u^{3 \gamma}, x^{0 \gamma}\right) \mathcal{S}_{13}\left(u^{3 \gamma}, w^{-\gamma}\right) \mathcal{S}_{23}\left(x^{0 \gamma}, w^{-\gamma}\right) \sim g^{-2} .
\end{aligned}
$$

According to (2.12) this could have been of order $g^{-3}$ when the physical magnon is a boson. What is more, the above relation can be generalized to an arbitrary number of physical magnons on the $0 \gamma$ edge:

$$
\begin{gathered}
\mathcal{S}_{12}\left(u^{3 \gamma}, v^{+\gamma}\right)\left[\prod_{j}^{\rightarrow} \mathcal{S}_{1 j}\left(u^{3 \gamma}, x_{j}^{0 \gamma}\right)\right]\left[\prod_{j}^{\rightarrow} \mathcal{S}_{2 j}\left(v^{+\gamma}, x_{j}^{0 \gamma}\right)\right] \sim g^{-2}, \\
{\left[\prod_{j}^{\rightarrow} \mathcal{S}_{1 j}\left(u^{3 \gamma}, x_{j}^{0 \gamma}\right)\right] \mathcal{S}_{12}\left(u^{3 \gamma}, w^{-\gamma}\right)\left[\coprod_{j}^{\leftarrow} \mathcal{S}_{j 2}\left(x_{j}^{0 \gamma}, w^{-\gamma}\right)\right] \sim g^{-2}}
\end{gathered}
$$

\footnotetext{
${ }^{4}$ More precisely, processes where fermions are transmitted are always order 1 ; reflection and bosonfermion processes are generically of order $g^{-1 / 2}$, and processes with physical bosons give more negative powers of $g$. The precise scaling depends on the choice of the magnon basis, in particular on the fermionnormalisation $e^{i p / 4} \sqrt{i\left(x_{p}^{+}-x_{p}^{-}\right)}$[27]. In the conventional basis bosons scale worst, like $g^{-1}$, while there exists a basis where all physical-fermion processes are of order 1. Final results are basis-independent.
} 
where $\vec{\prod} \mathcal{S}_{a j}=\mathcal{S}_{a 1} \mathcal{S}_{a 2} \ldots$ and $\overleftarrow{\prod} \mathcal{S}_{a j}=\mathcal{S}_{a N} \mathcal{S}_{a, N-1} \ldots$. Similar expressions hold for physical magnons on the $2 \gamma$ and $4 \gamma$ edges, or indeed combining physical magnons of different types:

$$
\begin{aligned}
{\left[\prod_{i}^{\leftarrow} \mathcal{S}_{1 i}\left(u^{3 \gamma}, y_{i}^{2 \gamma}\right)\right][} & \left.\prod_{j}^{\leftarrow} \mathcal{S}_{1 j}\left(u^{3 \gamma}, x_{j}^{0 \gamma}\right)\right] \mathcal{S}_{12}\left(u^{3 \gamma}, w^{-\gamma}\right) \\
& \times\left[\prod_{j}^{\rightarrow} \mathcal{S}_{j 2}\left(x_{j}^{0 \gamma}, w^{-\gamma}\right)\right]\left[\prod_{i}^{\rightarrow} \mathcal{S}_{i 2}\left(y_{i}^{2 \gamma}, w^{-\gamma}\right)\right] \sim g^{-2}
\end{aligned}
$$

Additional twists. So far we have discussed the weak-coupling properties of Beisert's scattering matrix and of the hexagon scalar factor; since we are looking at individual scattering processes our result will not rely on supersymmetry-induced cancellations such as the ones that take place when computing the transfer matrix and therefore also carry over to twisted transfer matrices (where the twist is applied to the Cartan elements of the $s u(2 \mid 2)$ algebra). However, it has been argued [10, 23] that for the purpose of computing four- and higher-point functions the $\mathrm{S}$ matrix should be modified by inserting a " $Z$-marker twist", and averaging over different twists. This construction cannot be derived from first principles - e.g., from a twist of the S-matrix symmetries. It is however justified by demanding the integrability results to match with perturbation theory. As it seems to be an important ingredient for computing correlation functions it is worth considering it in some detail. The " $Z$-marker twist" effectively introduce novel length-changing effects which in multi-particle states manifest themselves as $e^{ \pm i p(u) / 2}$ factors; these appear in opposite ways on pairs of neighbouring hexagons. Such $e^{ \pm i p(u) / 2}$ factors are rather harmless for physical excitations but they might yield additional powers of $g$ when $u$ is in the mirror or anti-mirror region. They hence require a separate analysis. Firstly, we note that as they appear on pairs of hexagons they do not yield any overall factor of $g$ in physical correlators. Secondly, by direct inspection we have found that the estimate of the scaling of the $\mathrm{S}$ matrix in the various channels which we considered above is not affected by the markers; furthermore, the same is true for the multiple-scattering processes. As our arguments below will solely rely on those weak-coupling estimates, our conclusions will hold also when $Z$-marker twists are included.

\section{Positivity of hexagon perturbation theory}

Let us now derive an explicit formula for the order in $g \ll 1$ at which a given hexagon configuration starts to contribute to the perturbative expansion. We first consider the case where we have only mirror excitations on the three mirror edges; this is the relevant case when computing arbitrary correlation functions of half-BPS operators.

\subsection{Naïve estimate of 't Hooft-coupling order}

The contribution of a single hexagon (2.1) is a little simpler for BPS operators

$$
\sqrt{\mu_{\mathbf{u}} \mu_{\mathbf{v}} \mu_{\mathbf{w}}} e^{\frac{i}{2} \tilde{E}_{\mathbf{u}} \ell_{\mathbf{u}}+\ldots} e^{i \psi \mathbf{J}+\ldots}\left\langle\mathfrak{h} \mid \varnothing, \mathbf{u}^{3 \gamma}, \varnothing, \mathbf{v}^{\gamma}, \varnothing, \mathbf{w}^{-\gamma}\right\rangle=O\left(g^{p}\right) .
$$


This scales like $g^{p}$ when $g \ll 1$, where the order $p$ is, naïvely

$$
p_{\text {naïve }}=\sum_{i=1}^{3} N_{i}\left(1+\ell_{i}\right)+2 \sum_{i=1}^{3} \frac{N_{i}\left(N_{i}-1\right)}{2}-2 N_{1} N_{2}-2 N_{1} N_{3}-2 N_{2} N_{3},
$$

where we have introduced the short-hand notations

$$
|\mathbf{u}|=N_{1}, \quad|\mathbf{v}|=N_{2}, \quad|\mathbf{w}|=N_{3} .
$$

Let us see how the different terms arise. Firstly, the first term in eq. (3.2) is given by the contribution of "half the measure" which scales as $g^{1}$ for each mirror magnon, plus half of the contribution of the bridge lengths. Next, when scattering all particles on the $i$ th mirror edge among themselves, we have a total of $N_{i}\left(N_{i}-1\right) / 2$ processes, each contributing $g^{2}$. Finally, and herein lies the rub, when scattering magnons from different edges we get negative contributions, of order $g^{-2}$ for each scattering event. We can rewrite the naïve estimate (3.2) as

$$
\begin{aligned}
p_{\text {naïve }} & =\sum_{i=1}^{3} N_{i} \ell_{i}+\sum_{i=1}^{3} N_{i}^{2}-2 N_{1} N_{2}-2 N_{1} N_{3}-2 N_{2} N_{3} \\
& =\sum_{i=1}^{3} N_{i} \ell_{i}+\left(N_{1}-N_{2}\right)^{2}+N_{3}^{2}-2 N_{3}\left(N_{1}+N_{2}\right),
\end{aligned}
$$

which is clearly unbounded from below, for instance when $N_{1} \sim N_{2} \gg N_{3} \gg \ell_{i}$.

Improving the estimate. Bearing in mind the observation of eq. (2.13), we can get a better estimate for the scaling of a single hexagon, namely

$$
p=p_{\text {naïve }}+4 T\left(N_{1}, N_{2}, N_{3}\right),
$$

where $T\left(N_{1}, N_{2}, N_{3}\right)$ is the number of "triple" scattering events such as the ones of eq. (2.13). We are therefore interested in arranging the scattering in such a way as to maximise such triple scattering processes.

\subsection{Triple scattering processes}

It is convenient to introduce a diagrammatic representation of the scattering processes which we will consider. In practice, we have three sets of lines $\{\mathbf{u}\},\{\mathbf{v}\},\{\mathbf{w}\}$, which we want to rearrange by reversing their order,

$$
\left(u_{1}, \ldots u_{N_{1}}, v_{1}, \ldots v_{N_{2}}, w_{1}, \ldots w_{N_{3}}\right) \rightarrow\left(w_{N_{3}}, \ldots w_{1}, v_{N_{2}}, \ldots v_{1}, u_{N_{1}}, \ldots u_{1}\right) .
$$

For the purpose of counting the triple-intersection of lines of type $u, v$ and $w$ the rearrangement within each set $\{\mathbf{u}\},\{\mathbf{v}\},\{\mathbf{w}\}$ is irrelevant. Hence we can consider the slightly simpler process

$$
\left(u_{1}, \ldots u_{N_{1}}, v_{1}, \ldots v_{N_{2}}, w_{1}, \ldots w_{N_{3}}\right) \rightarrow\left(w_{1}, \ldots w_{N_{3}}, v_{1}, \ldots v_{N_{2}}, u_{1}, \ldots u_{N_{1}}\right) .
$$




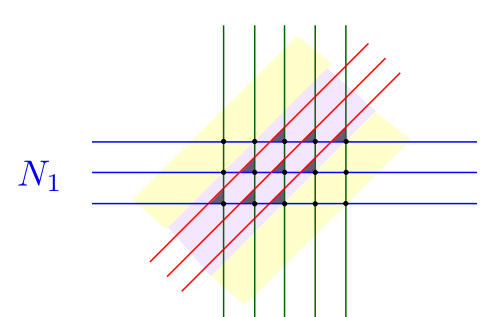

$N_{3}$

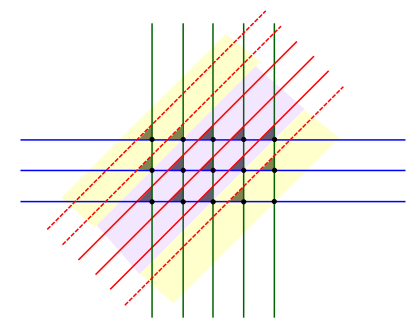

$N_{3}$

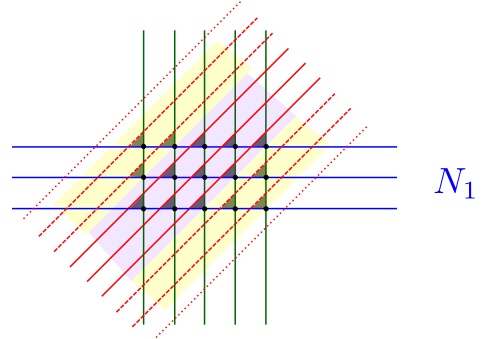

$N_{3}$

case-I

case-II

case-III

Figure 2. Diagrammatic representation of scattering processes involving three sets of mirror magnons labelled by $N_{1}, N_{2}$ and $N_{3}$, corresponding to particles of type $\mathbf{u}, \mathbf{v}$ and $\mathbf{w}$, respectively. We start by drawing the lines corresponding to $N_{1}$ and $N_{3}$ (assuming for definiteness $N_{1} \leq N_{3}$ ) and then place, one by one, the diagonal lines corresponding to $N_{2}$ in such a way as to maximise the triple scattering of eq. (2.13) - the dark triangles. As detailed in the main text, we see three distinct behaviours depending on how many diagonal lines we need to place: for the first few, which we place in the purple region (see left panel), we get a constant number of triangles per diagonal line. After a certain point (yellow region, middle panel), the number of triangles decreases as we add lines. Eventually, adding more diagonal lines does not yield any new triangle (right panel).

We have represented such a scattering process in figure 2 . We start by drawing $N_{1}$ horizontal and $N_{3}$ vertical lines, which we can do unambiguously. For definiteness, we shall assume

$$
N_{1} \leq N_{3}
$$

Next, we can add one by one the $N_{2}$ lines corresponding to $\mathbf{v}$, which should go from southwest to north-east of the figure. This can be done in several equivalent ways, owing to the Yang-Baxter equation. We want to do so in such a way as to maximise the triple scattering discussed above, which here results in a triangular vertex (see also the figure). It is convenient to distinguish three cases, depending on the value of $N_{2}$.

Case I. Looking at the leftmost panel in figure 2, we imagine adding a single diagonal line. Clearly we obtain at most as many triangles as we have horizontal lines, i.e. $N_{1}$ triangles - recall that $N_{1} \leq N_{3}$. We can go on adding diagonal lines as long as we saturate the purple area in the region; indeed we can do so $N_{3}-N_{2}+1$ times at most. Therefore, we have that

$$
T\left(N_{1}, N_{2}, N_{3}\right)=N_{2} N_{1}, \quad N_{2} \leq N_{3}-N_{1}+1 .
$$

Case II. Let us keep adding diagonal lines, looking this time at the middle panel of figure 2. To maximise the number of triangles, we add diagonal lines just above or just below those we had drawn above. We have two choices with $\left(N_{1}-1\right)$ triangles (above and below), two choices with $\left(N_{1}-2\right)$ triangles, and so on. Adding one line at the time, we get the sequence

$$
N_{1}-1, N_{1}-1, N_{1}-2, N_{1}-2, N_{1}-3, N_{1}-3, \ldots
$$


which reaches zero in $2 N_{1}$ steps. Therefore, as long as $N_{2} \leq N_{3}-N_{1}+1+2 N_{1}=N_{3}+N_{1}+1$, the number of new triangles (in the yellow region of the figure) is given by the sum

$$
\sum_{j=1}^{K}\left[N_{1}-\frac{\left(2 j+1-(-1)^{j}\right)}{4}\right]
$$

which can run up at most $K=N_{2}-\left(N_{3}-N_{1}+1\right)$ steps. The slightly quirky summand just reproduces the sequence (3.10). Bearing in mind that we have $N_{1}\left(N_{3}-N_{1}+1\right)$ triangles in the purple region, with a little algebra we find that the total number of triangles is at most

$$
\begin{array}{r}
T\left(N_{1}, N_{2}, N_{3}\right)=\frac{N_{1} N_{2}+N_{1} N_{3}+N_{1} N_{2}}{2}-\sum_{i=1}^{3} \frac{N_{i}^{2}}{4}+\frac{1+(-1)^{\sum_{i} N_{i}}}{8}, \\
\text { for } \quad N_{3}-N_{1}+1<N_{2} \leq N_{3}+N_{1}+1 .
\end{array}
$$

We shall later comment on the fractional coefficients; for the moment, suffice is to say that by construction this number is an integer despite of its complicated appearance.

Case III. Finally, we can keep adding diagonal lines like in the rightmost panel of figure 2. This does not generate any new triangles. Therefore, we can just use the previous estimate, taking the limit of the sum (3.11) to be the maximal allowed value $M=2 N_{1}$. Then we find that

$$
T\left(N_{1}, N_{2}, N_{3}\right)=N_{3} N_{1}, \quad N_{3}+N_{1}+1<N_{2},
$$

as we could have expected from the diagrammatical representation: we simply have one triangle for each vertex between horizontal and vertical lines.

\subsection{Improved estimate of 't Hooft-coupling order}

Armed with these estimates, it is not difficult to see case by case at which order $O\left(g^{p}\right)$ a digram with $\left(N_{1}, N_{2}, N_{3}\right)$ mirror particles contributes.

Case I. Here we have

$$
p=\sum_{i=1}^{3} N_{i} \ell_{i}+\left(N_{3}-N_{1}-N_{2}\right)^{2}, \quad N_{2} \leq N_{3}-N_{1}+1,
$$

which is positive semi-definite regardless of the values of $\ell_{i}$.

Case II. In the second case we are left with

$$
p=\sum_{i=1}^{3} N_{i} \ell_{i}+\frac{1+(-1)^{\sum_{i} N_{i}}}{2}, \quad N_{3}-N_{1}+1<N_{2} \leq N_{3}+N_{1}+1 .
$$

Note that the fact that $p$ is not necessarily even is due to the fact that we are considering a single hexagon, and have split the measure and bridge length as in eq. (2.1). At weak coupling, we expect the expansion to be in powers of $g^{2}$ when all hexagons are glued together. 
Case III. Similar to case I we have

$$
p=\sum_{i=1}^{3} N_{i} \ell_{i}+\left(N_{2}-N_{1}-N_{3}\right)^{2}, \quad N_{2}>N_{3}+N_{1}+1 .
$$

Infinite chains of mirror magnons. Notice that while the present estimate makes $p$ bounded from below, unlike the orginal naïve estimate (3.2), it is still possible to have infinitely many processes contributing at a given order. This can be achieved by setting e.g. $N_{3}$ to a fixed values and varying $N_{1}$ and $N_{2}$, provided that both bridge lengths $\ell_{1}, \ell_{2}$ vanish. More generally, it is only possible for infinitely many magnons to contribute at a given order of the small-coupling expansion of a single hexagon when two bridge lengths vanish. This situation may well appear when computing correlators, and is all the more frequent when considering non-planar topologies, see e.g. ref. [11]. We could not find a way to rule out such processes on general grounds, though it is possible to make some progress when considering particular correlators. In section 4 we will look in detail at how these processes appear in the four-point functions of BPS operators. We will see that it is still possible to use the bound we have obtained here, along with some observations from field theory, to reduce the computation to a finite set of mirror exchanges.

\subsection{Physical magnons}

From eq. (2.12) we see that, as long as physical magnons are given by fundamental fermionic excitations - for instance, for non-BPS operators in the $s l(2)$ sector - our estimates from the previous section apply immediately since no negative powers of $g$ emerge from physicalmirror scattering. Things are a little more involved if we allow for bosonic excitations. Consider for instance a hexagon containing a set of physical particles $\{\mathbf{x}\}$ in the $s o(6)$ sector, so that the hexagon is schematically

$$
\sqrt{\mu_{\mathbf{u}} \mu_{\mathbf{v}} \mu_{\mathbf{w}}} e^{\frac{i}{2} \tilde{E}_{\mathbf{u}} \ell_{\mathbf{u}}+\ldots} e^{i \psi \mathbf{J}+\ldots}\left\langle\mathfrak{h} \mid \varnothing, \mathbf{u}^{3 \gamma}, \varnothing, \mathbf{v}^{\gamma}, \mathbf{x}, \mathbf{w}^{-\gamma}\right\rangle=O\left(g^{p}\right) .
$$

The contribution of the $M=|\mathbf{x}|$ physical so(6) magnons naïvely would be

$$
p \rightarrow p-M N_{2} \quad \text { naïvely, }
$$

which once again would yield an estimate which is unbounded from below as $N_{2}$ grows. Again we can improve our estimate, this time using the triangle identity (2.15).

Improved estimate. Consider the picture in figure 3. Here we have further decorated a graph such as the one of figure 2 by adding a new set of lines, denoted by $M$, and corresponding to $M$ physical magnons. We are focussing here on what we called case III in figure 2, which happens when

$$
N_{2}>N_{1}+N_{3}+1
$$

The reason is that the only runaway behaviour can arise as $N_{2} \rightarrow \infty$, which constrains us to this scenario. We have already maximised the number of $u-v-w$ triangles following the logic explained above. Now we want to maximise the number of multiple scattering 

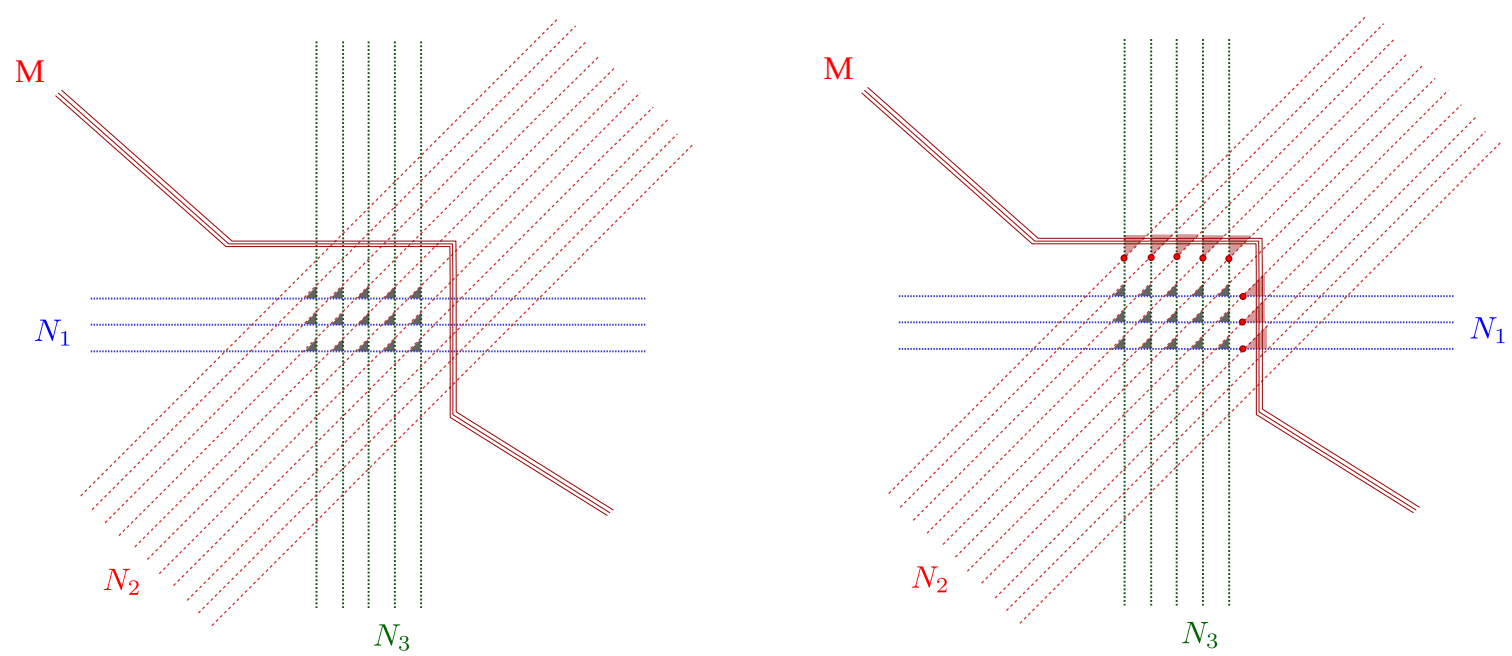

Figure 3. We draw again the scattering processes of figure 2, focussing on case III and adding $M$ physical magnons. To guide the eye, we have dotted the lines corresponding to mirror magnons. On the left, we have $M N_{2}$ scattering events between physical magnons and magnons on the opposing edge. On the right panel, we highlight the "cones" emanating from the scattering of a mirror particle on the opposite edge with one from the adjacent edge; this is the vertex of the cone, and it is marked by a dot. There are clearly $N_{1}+N_{3}$ such cones.

events as in eq. (2.15). These can be thought of as "cones" emanating either from a $u-v$ triangle or $v-w$ vertex, and involving an arbitrary number of physical particles $x$. From the figure we see that there are $N_{1} u-v$ vertices and $N_{3} v-w$ vertices, bearing (3.19) in mind, for a total of $N_{1}+N_{3}$ cones. Furthermore, each cone contains $M$ scattering events between physical magnons and mirror magnons on the opposite edge. Hence, our naïve estimate is improved by a factor of $M\left(N_{1}+N_{3}\right)$. All in all,

$$
p \rightarrow p-M\left(N_{2}-N_{1}-N_{3}\right),
$$

so that in presence of $M$ physical magnons

$$
p=\sum_{i=1}^{3} N_{i} \ell_{i}+\left(N_{2}-N_{1}-N_{3}\right)\left(N_{2}-N_{1}-N_{3}-M\right) .
$$

Hence $p$ is still bounded from below, even if its minimum value (as $N_{2}$ varies) can become negative. In particular, we find that

$$
p_{\min }=N_{1}\left(\ell_{1}+\ell_{2}\right)+N_{3}\left(\ell_{3}+\ell_{2}\right)-\frac{M^{2}-2 M}{4},
$$

which is bounded from below for fixed $M$ (i.e., for any given physical state). Similar bounds can be derived for more general configurations of physical magnons, see appendix B.

Application to correlators of non-BPS operators. Our estimate for $p$ remains bounded from below and is only slightly worsened by the inclusion of physical magnons if those sit in the so(6) sector. Moreover, we might expect the estimate to further improve 
when restricting to a particular topology for the correlator - as it is the case for three-point functions [21]. This could arise both from gluing different hexagons, from summing over the partitions of physical magnons, and from exploiting the fact that physical states satisfy the Bethe ansatz equations. It is still a bit surprising that $s o(6)$ physical excitations behave so differently from other magnons in the physical-mirror scattering. This is essentially due to "string-frame" factors $[6,27]$ in the $s u(2 \mid 2)$ scattering matrix, which in the mirror kinematics affects the $g$-scaling. While at the asymptotic level the hexagon formalism can be equivalently formulated either in the spin-chain or string frames [8], things are a little muddier when looking at wrapping corrections. In particular, it appears that additional Z-markers, which mimic the frame factors, need to be taken into account when considering mirror magnons in higher-point correlation functions [10, 23]. This procedure is, at least at this stage, only justified by a posteriori matching with perturbation theory. As these checks have so far focussed on non-protected operators in the $s l(2)$ sector only, it would be very interesting to derive correlation functions of operators from the $s u(2)$ subsector via the hexagon approach, and understand how these match the field-theory results, and whether perhaps some additional $\mathrm{Z}$ markers modify our counting - for instance making $p_{\min }$ positive definite. In any case, we stress that our estimate - based on the current understanding of virtual-magnon computations in the hexagon formalism - is sufficient to exclude runaway behaviours even in presence of physical magnons.

\section{Application to planar BPS four-point functions}

In this section we apply our results above to the computation of a particular correlation function; we will especially focus on the issue of infinite chains of mirror magnons. Let us consider a planar four-point function of half-BPS operators

$$
\left\langle\mathcal{O}_{k_{1}}\left(x_{1}\right) \mathcal{O}_{k_{2}}\left(x_{2}\right) \mathcal{O}_{k_{3}}\left(x_{3}\right) \mathcal{O}_{k_{4}}\left(x_{4}\right)\right\rangle, \quad \mathcal{O}_{k}=\operatorname{Tr}\left(\hat{Z}^{k}\right),
$$

where the six real scalars $\phi^{I}$ of $\mathcal{N}=4 \mathrm{SYM}$ are rewritten in the linear combination $\hat{Z}=\sum_{I=1}^{6} \phi^{I} Y^{I}$, with $Y$ a complex null vector. Two fields $\hat{Z}_{i}\left(x_{i}\right)$ and $\hat{Z}_{j}\left(x_{j}\right)$ have the free propagator

$$
\Pi_{i j}=\frac{Y_{i} \cdot Y_{j}}{\left(x_{i}-x_{j}\right)^{2}} .
$$

As proposed in refs. $[9,10]$, to compute such a four-point function by hexagon tessellations we start by listing all propagator combinations with the right conformal weights $k_{1}, k_{2}, k_{3}, k_{4}$. These are simply

$$
\left\{\Pi_{12}^{\ell_{12}} \Pi_{13}^{\ell_{13}} \Pi_{14}^{\ell_{14}} \Pi_{23}^{\ell_{23}} \Pi_{24}^{\ell_{24}} \Pi_{34}^{\ell_{34}}: \quad k_{i}=\sum_{j} \ell_{i j}\right\} .
$$

Notice that the number of propagator $\ell_{i j}$ in the strand going from point $i$ to point $j$ is exactly the bridge length which we discussed in section 3 , and which is a crucial part of our final estimate for $p$, see eq. (3.5) and below. We are interested in planar graphs, i.e. graphs that can be drawn on a sphere without intersecting propagators; not all assignments of $\ell_{i j}$ 

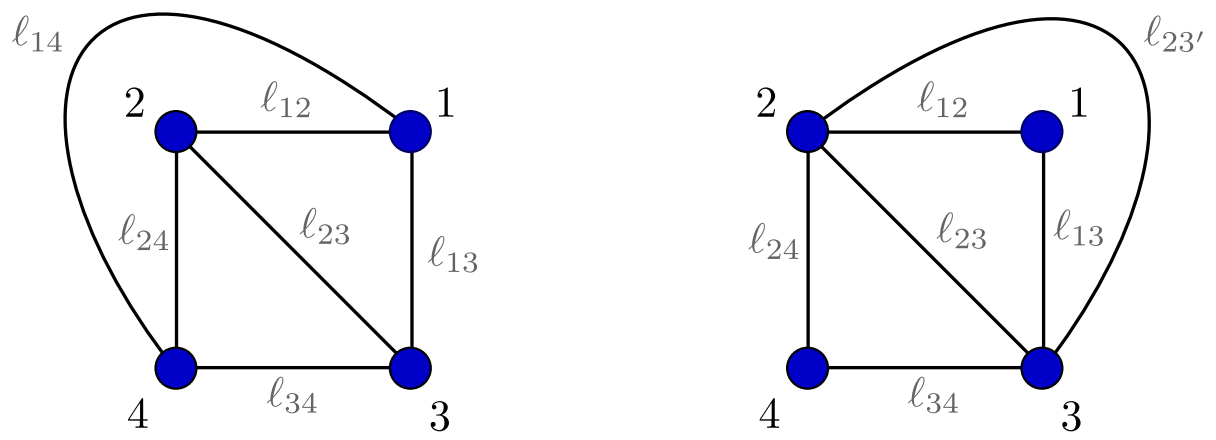

Figure 4. Hexagon tessellations for planar four-point functions. The four points lie on a sphere which we represent on the plane. Solid lines represent strands of propagators, see eq. (4.3), which dictate in which way to tessellate the diagram. Up to relabeling the points 1234 these are the only planar tree-level diagrams $[9,10]$. Planarity restricts the $\ell_{i j}$ that can be non-zero to those in the figure. Still, it is possible that a subset of the solid lines in the figure have $\ell_{i j}=0$, as we shall see below.
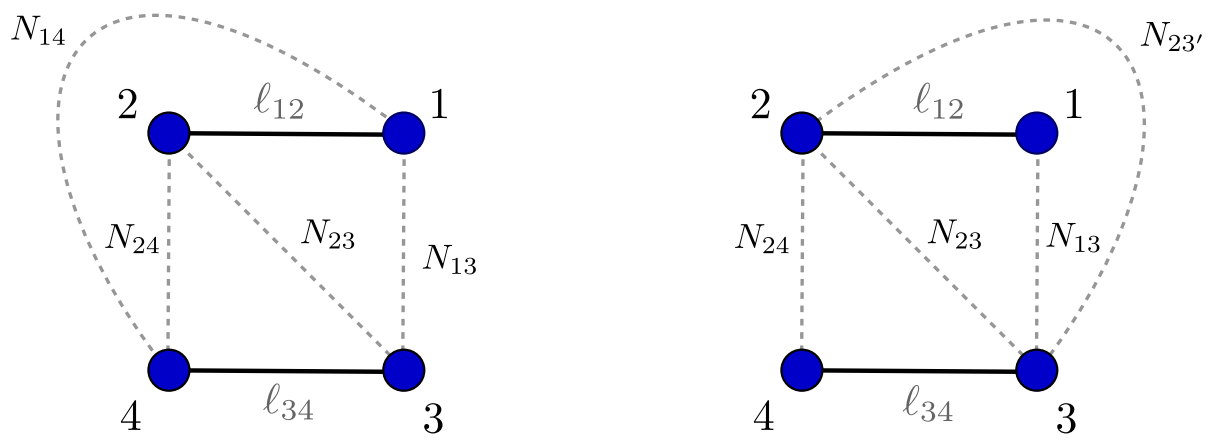

Figure 5. Four-point functions with four vanishing bridge lengths. The resulting hexagon tessellations allow for infinite chains of mirror magnons (along the zero-length bridges) already at order $g^{0}$.

give rise to such graphs. It is easy to see this diagrammatically. In figure 4 we show the choices of $\ell_{i j}$ that give rise to planar graphs and the relative hexagon tessellation. For any allowed choice of propagators resulting in such a planar topology from the set (4.3), we have to decorate the figure with all mirror magnons allowed by our estimates of section 3 , up to the order in $g^{2}$ in which we are interested.

Notice that already at the lowest orders in $g \ll 1$ this can involve an infinite chain of mirror particles. More specifically, this can happen when sufficiently many mirror edges have vanishing bridge length. Consider figure 5, and notice that e.g. in the left diagram

$$
\ell_{24}=\ell_{23}=\ell_{13}=\ell_{14}=0
$$

Let us now estimate the order $g^{p}$ at which this graph contriutes, restricting to the case where there are no mirror magnons on the non-zero length bridges $\left(N_{12}=N_{34}=0\right)$, which 
turns out to be the key example. Then we have ${ }^{5}$

$$
\begin{aligned}
p & =2\left(N_{24}^{2}+N_{23}^{2}+N_{13}^{2}+N_{14}^{2}-N_{24} N_{23}-N_{23} N_{13}-N_{13} N_{14}-N_{12} N_{24}\right) \\
& =\left(N_{24}-N_{23}\right)^{2}+\left(N_{23}-N_{13}\right)^{2}+\left(N_{13}-N_{14}\right)^{2}+\left(N_{14}-N_{24}\right)^{2} .
\end{aligned}
$$

In the first line, the quadratic contributions come from the interaction of magnons on the same mirror edge (including the mirror measure), while the bilinear terms come from scattering magnons on different edges within the same hexagon. The expression on the second line makes it transparent that we can have infinitely many mirror magnons contributing at given orders of perturbation theory $g^{p}$ by tuning the values of $N_{i j}$. For instance, this happens when $N_{24}=N_{23}=N_{13}=N_{14}=N$ and we let $N=1,2, \ldots$ This process should contribute already at tree-level! Going to slightly higher orders in $g^{2}$, more trouble appears. Take for instance $N=N_{24}=N_{23}=N_{13}=N_{14}-1$ : all these processes would appear at order $g^{2}$; similarly, we find more such "chains" at higher orders. Moreover, depending on the loop order which interests us we may also need to consider more complicated processes. For instance, by putting a single mirror magnon on edge $12\left(N_{12}=1\right)$ we obtain

$$
p=2 \ell_{12}+\left(N_{24}-N_{23}\right)^{2}+\left(N_{23}-N_{13}+1\right)^{2}+\left(N_{13}-N_{14}\right)^{2}+\left(N_{14}-N_{24}+1\right)^{2},
$$

where it was crucial to use the improved estimate of section 3 for $p$ to be positive. We see that such processes give infinite chains of magnons starting from order $g^{2 \ell_{12}}$. Clearly the same can be done on edge 34 .

What is the physical interpretation of these processes? Since they only stem from disconnected tree-level graphs, they seem quite pathological; however, it is not obvious that we can discard them. The tree-level graph should indeed be trivial, so that the $g^{0}$ contribution from this infinite chain of magnons vanish. However, at higher orders, we might imagine that introducing mirror particles corresponds to inserting virtual gluon lines in field theory [11], which might give a connected four-point graph. At one loop, it has been shown that the hexagon prediction without any infinite chain matches the field theory result [10], so it seems that these vanish at that order too. What about higher loops?

As such infinite chains can hardly be evaluated directly, to address this problem it is useful to notice that there is some redundancy in the hexagon formulation, which results in linear identities among apparently unrelated mirror-magnon processes. Let us denote the topologies appearing in the left and right panel in figure 4 as $G_{1}, G_{2}$, respectively. For each of these topologies we let $\underline{\ell}, \underline{n}$ be the tuples of six edge widths and the six corresponding occupation numbers. For topology 1 we have $\underline{\ell}=\left(\ell_{12}, \ell_{24}, \ell_{34}, \ell_{13}, \ell_{23}, \ell_{14}\right)$ and for topology 2 we set $\underline{\ell}=\left(\ell_{12}, \ell_{24}, \ell_{34}, \ell_{13}, \ell_{23}, \ell_{23^{\prime}}\right)$; the tuple $\underline{n}$ is similarly defined. Last, let us factor the contribution of a tree (or skeleton) graph with edge widths $\underline{\ell}$ dressed by $\underline{n}$ mirror magnons a

$$
G_{I}^{\underline{\ell} \underline{\underline{n}}}=F_{I}^{\underline{\ell}, \underline{n}} \Pi_{I}^{\ell}, \quad I=1,2,
$$

where $\Pi \frac{\ell}{I}$ is the product of free propagators and $I$ denotes the topology. The function $F_{I}^{\ell} \underline{\underline{n}}$ is then a function of conformal invariants. By way of example, the integral int 14 in [10]

\footnotetext{
${ }^{5}$ Notice that here there is no possibility to have "triple scattering events" (see section 3 ) and our estimate for the 't-Hooft coupling order $g^{p}$ coincides with the naïve one (3.2).
} 
arises from $F_{2}^{\underline{\ell}, \underline{n}}$ with $\underline{n}=(0,0,0,0,0,1)$. Linear relations among different $F_{\bar{I}}^{\underline{\ell}, \underline{n}}$ arise in five ways:

1. When several $\ell_{i j}$ 's vanish it is possible to embed the same tree-level graph into different tessellations. It is natural to assume that all such embeddings are equivalent. This condition, which we call embedding invariance, has been checked on several examples so far [9-11]. For illustration, we can consider a graph where the only non-vanishing lengths are those corresponding to $\ell_{12}, \ell_{24}, \ell_{43}, \ell_{31}$ which are the first four entries of $\underline{\ell}$ in our convention. Clearly such a graph can be embedded on $G_{1}$ as well as on $G_{2}$. Hence for any such $\underline{\ell}$, embedding invariance yields

$$
\sum_{\underline{n}_{1} \geq 0} F_{1}^{\underline{\ell}, \underline{n}_{1}}=\sum_{\underline{n}_{2} \geq 0} F_{2}^{\underline{\ell}, \underline{n}_{2}}
$$

In practice, we can expand both sides in $g \ll 1$ to a given order and truncate the infinite sums over $\underline{n}_{1}, \underline{n}_{2}$ accordingly. There is such an equation for each pair of embeddings of any given tree graph.

2. The hexagon formula (3.1) depends only on the lengths of the bridges which contain at least one mirror magnon [10]; hence diagrams with no magnons on edge $i j$ give identical integrals for any $\ell_{i j}$. We call this property forgetfulness of the hexagons:

$$
F^{(\ldots, \ell, \ldots),(\ldots, 0, \ldots)}=F^{(\ldots, 0, \ldots),(\ldots, 0, \ldots)} .
$$

In particular, at tree level $F^{\underline{l}, \underline{0}}=1$.

3. In four dimensions, conformal functions of four points on Minkowski space depend on two cross ratios, which we can write e.g. as

$$
z \bar{z}=\frac{x_{12}^{2} x_{34}^{2}}{x_{13}^{2} x_{24}^{2}}, \quad(1-z)(1-\bar{z})=\frac{x_{14}^{2} x_{23}^{2}}{x_{13}^{2} x_{24}^{2}} .
$$

Additionally, $F_{\bar{I}}^{\underline{\ell} \underline{\underline{n}}}$ depends on two similar cross ratios on the four-dimensional internal space, denoted as $\alpha, \bar{\alpha}$. Now, the simultaneous exchange of two pairs of distinct points $i \leftrightarrow j, k \leftrightarrow l$ leaves the cross ratios invariant, as it can be seen from the right-hand side of eq. (4.10). On the other hand, the double permutations act non-trivially on the topologies $G_{1}, G_{2}$. Consider $G_{2}$ under the point permutation $\sigma=(14)(23)$ : we have

$$
\underline{\ell}=\left(\ell_{12}, \ell_{24}, \ell_{34}, \ell_{13}, \ell_{23}, \ell_{23^{\prime}}\right) \rightarrow \underline{(\sigma \circ \ell)}=\left(\ell_{34}, \ell_{13}, \ell_{12}, \ell_{24}, \ell_{23}, \ell_{23^{\prime}}\right)
$$

As a consequence we get

$$
F_{1}^{l, \underline{n}}=F_{1}^{(\sigma \circ \ell)}, \underline{(\sigma \circ n)},
$$

as well as a similar condition for $F_{2}$. Graphically this corresponds to a symmetry of the diagram under simultaneous rotations by an angle of $\pi$ around both symmetry axes. Similarly, the two other double point exchanges (12)(34) and (13)(24) map the 
diagram $G_{2}$ onto a topology with two 14 diagonals. ${ }^{6}$ Similar considerations apply to $G_{1}$. Note that we are working in terms of the $F_{\bar{I}}^{\ell, \underline{n}}$ functions, as they are the ones expressed in terms of the cross-ratios; the propagator factor $\Pi_{I}^{\ell} \underline{\underline{\ell}}$ transform covariantly in general.

4. It is also known that certain four-point functions obey non-renormalisation theorems [30-32]; this happens for of "extremal" and "sub-extremal" correlators, in which the lengths of the four operators obey $L_{1}=L_{2}+L_{3}+L_{4}$ and $L_{1}=L_{2}+L_{3}+L_{4}-2$, respectively. For every correlator one will list the tree level diagrams allowed by the charges of the outer operators and proceed to compute them by tessellating the diagrams as usual. The resulting sum of $F_{\bar{I}}^{\underline{\ell}} \underline{\underline{n}}$ terms has to vanish.

5. Furthermore, we can match correlators on explicit results [33] for the $\mathrm{SU}(N)$ gauge group; this gives constraints for the mirror-magnon processes that appear in more general situations, too. The formulae in [33] contain only a few conformal four-point integrals, which can be eliminated from a sufficiently large set of distinct four-point functions leaving linear constraints on the $F_{\bar{I}}^{\ell, \underline{n}}$ terms.

In this spirit, we have considered all possible correlators of half-BPS operators with the length of each of the four operators ranging from 2 to 7 , at one- and two-loop level. Consider a diagram where an "infinite chains" of mirror magnons appears. It is convenient to treat such a chain as if it were a single term in our $F_{\bar{I}}^{\ell} \underline{\underline{n}}$ expansion. Up to a given loop order, we have then a finite number of $F_{\bar{I}}^{\underline{\ell}} \underline{n}$ functions appearing - though we understand that some of them really collect a whole infinite chain of mirror magnons. In practice, up to two loops and for the correlation functions we described this yields a few thousand $F_{\bar{I}}^{\ell, \underline{n}}$ terms. Spelling out the various constraints arising in the way outlined above, we find a similar number of equations. A linear solver can easily deal with the equations, although the solution is too bulky to report it here.

Instead, let us comment on the features of infinite mirror-magnon chains that this approach reveals at order $g^{4}$. Firstly and importantly, we find that infinite chains of mirror magnons of the type of eq. (4.6) vanish also at order $g^{4}$ (we might have expected such processes to appear when $\ell_{12}=2$ or $\ell_{34}=2$ ). As for the chain of eq. (4.5), it does not vanish immediately, but it can be recast as a sum of finitely many mirror processes (involving at most three mirror magnons), so that it can be evaluated directly. It is, of course, somewhat disappointing that these considerations, much like the ones at order $g^{2}$ and $g^{0}$ do not stem only from the internal consistency of the hexagon formalism, but require input from field theory.

\section{Conclusions and outlook}

In this paper we have improved on the naïve bound for the 't Hooft-coupling scaling of a hexagon form factor with a set of $N_{1}, N_{2}, N_{3}$ mirror magnons. That would have been

\footnotetext{
${ }^{6}$ This latter mechanism was used in ref. [29] to obtain what in that article was dubbed a magic identity between conformal integrals.
} 
order $g^{p}$ with

$$
p_{\text {naïve }}=\sum_{i=1}^{3} N_{i} \ell_{i}+\left(N_{1}-N_{2}\right)^{2}+N_{3}^{2}-2 N_{3}\left(N_{1}+N_{2}\right),
$$

which is unbounded from below as $N_{1}, N_{2}$ and $N_{3}$ grow - signalling an apparent breakdown of the weak-coupling perturbation theory for the hexagon form-factor program and flying in the face of established perturbative results such as those of refs. [9-11, 23]. We have shown that such naïve bound can be improved and is non-decreasing as $N_{1}, N_{2}$ and $N_{3}$ grow. Similar considerations apply in the presence of physical magnons.

However, even our improved formula allows for infinite "chains" of mirror particles at finite order in $g^{p}$. They appear only when two out of three bridge lengths $\ell_{i}$ vanish on a given hexagon. In the case of four-point functions, which we investigated at some length in section 4, this is what happens for tessellations built from disconnected treelevel graphs. While we could not find an argument to argue these chains away within the hexagon formalism, we have observed that in practice (taking into account field theory considerations) we do not need to compute such an infinite sum of terms; we can instead trade them for a finite sum of mirror-magnon contributions, at least up two loops in field theory - order $g^{4}$. It is not hard to believe that such infinite chains of magnons might simplify drastically: viewing the resulting sum-integrals as Mellin representations [10] one might e.g. expect that a chain of gluings over zero-width edges can be simplified as in Barnes' lemma. ${ }^{7}$ Still it would be very interesting to understand from first principles whether such infinite chains of magnons should be disregarded and why.

Having a systematic understanding of the dynamics of mirror magnons is an important first step towards the summation of finite-size corrections to obtain a truly non-perturbative framework, perhaps along the lines of the mirror thermodynamic Bethe ansatz formalism for two-point functions. It is not obvious what is the best context to tackle this important and challenging problem. While the hexagon formalism is established only for $\mathrm{AdS}_{5} / \mathrm{CFT}_{4}$, where the integrability machinery is best developed, it is interesting to note that in integrable $\mathrm{AdS}_{3} / \mathrm{CFT}_{2}$ [34] there exist models where wrapping effects drastically simplify $[35,36]$ - in fact, they vanish for two-point functions - and where closed formulae exist for some correlation functions owing to worldsheet (Wess-Zumino-Witten) techniques. This might also be an excellent arena to test the hexagon program.

Let us also remark that the same reasoning that allowed us to circumvent infinite sums of mirror magnons (as explained in section 4) also leads us to a number of interesting observations which would greatly simplify the computation of the four-BPS correlator at order $g^{4}$. For instance, a rather problematic class of diagrams is the one which we can obtain from the disconnected ones of figure 5 by setting e.g. $\ell_{13}=1$; we call these "sausage" graphs. There, infinite chains of mirror magnons do not appear but we find a large number of possible processes: at order $g^{2}$ we should consider up to four mirror magnons, while at order $g^{4}$ we should consider up to eight mirror magnons. Such a proliferation is worrying, as it makes the hexagon formalism very cumbersome. On the other hand, using the cri-

\footnotetext{
${ }^{7}$ B.E. thanks Benjamin Basso for a discussion on related matters.
} 
teria 1.-5. of the section above, we can see that there are numerous cancellations, which happen graph-by-graph for processes involving different numbers of mirror magnons. In particular, processes involving four magnons are suppressed and only appear at order $g^{4}$, and processes involving five or more magnons only appear at order $g^{6}$. It is interesting to observe that this cancellation is also compatible with a further field-theory inspired constraint, that is the maximum transcendentality of a correlator increases with the order of $g^{2}$. Technically, this order appears in the hexagon formalism from the highest-pole order in the integrand of mirror processes. This pole comes ${ }^{8}$ from the mirror measure and bridgelength contribution. On these grounds, we would expect that mirror integrals related to edges with length $\ell$ only contribute at order $g^{2 \ell+2}$ or higher, as it is indeed the case for sausage graphs. In fact, this principle may be taken as an additional constraint in future hexagon-form-factor computations.

\section{Acknowledgments}

BE is supported by DFG "eigene Stelle" Ed 78/4-3. MdL was supported by SFI and the Royal Society for funding under grant UF160578. D. le Plat acknowledges support by the Stiftung der Deutschen Wirtschaft. AS's work is partially supported by the NCCR SwissMAP, funded by the Swiss National Science Foundation; he also acknowledges support by the ETH "Career Seed Grant" no. 0-20313-17.

\section{A Algorithm for triple mirror scattering}

In this section we give an alternative derivation of the number of triple scattering events $T\left(N_{1}, N_{2}, N_{3}\right)$ discussed in section 3. This derivation is based on an algorithmic derivation of the scattering process that maximize the number of triangles. In order to introduce the notation and explain the idea, we first consider a simple example with $\left(N_{1}, N_{2}, N_{3}\right)=$ $(3,3,3)$. In what follows, we will denote the configuration $\left(N_{1}, N_{2}, N_{3}\right)$ by

$$
\underbrace{1 \cdots 1}_{N_{1}} \underbrace{2 \cdots 2}_{N_{2}} \underbrace{3 \cdots 3}_{N_{3}} \equiv(1)^{N_{1}}(2)^{N_{2}}(3)^{N_{3}}
$$

We expect to have $T(3,3,3)=7$ from the previous section. Here we demonstrate how this can be realized. We start with the configuration

$$
111222333 .
$$

According to the hexagon form factor prescription, we move the 1 's to the right and 3 's to the left by scattering adjacent magnons by the $S$-matrix. The process is as follows

$$
\begin{aligned}
& 111222333 \longrightarrow 112[123] 233 \longrightarrow 1[123] 2[123] 3 \\
\longrightarrow & 132[123] 213 \longrightarrow 132321213 \longrightarrow[123] 321[123] \\
\longrightarrow & 321321321 \longrightarrow 323[123] 121 \longrightarrow 323321121
\end{aligned}
$$

\footnotetext{
${ }^{8}$ This can be seen most readily by rewriting the integrand by taking partial fractions, like in refs. [20, 21], and observing that partial fractioning preserves the order of the leading pole.
} 


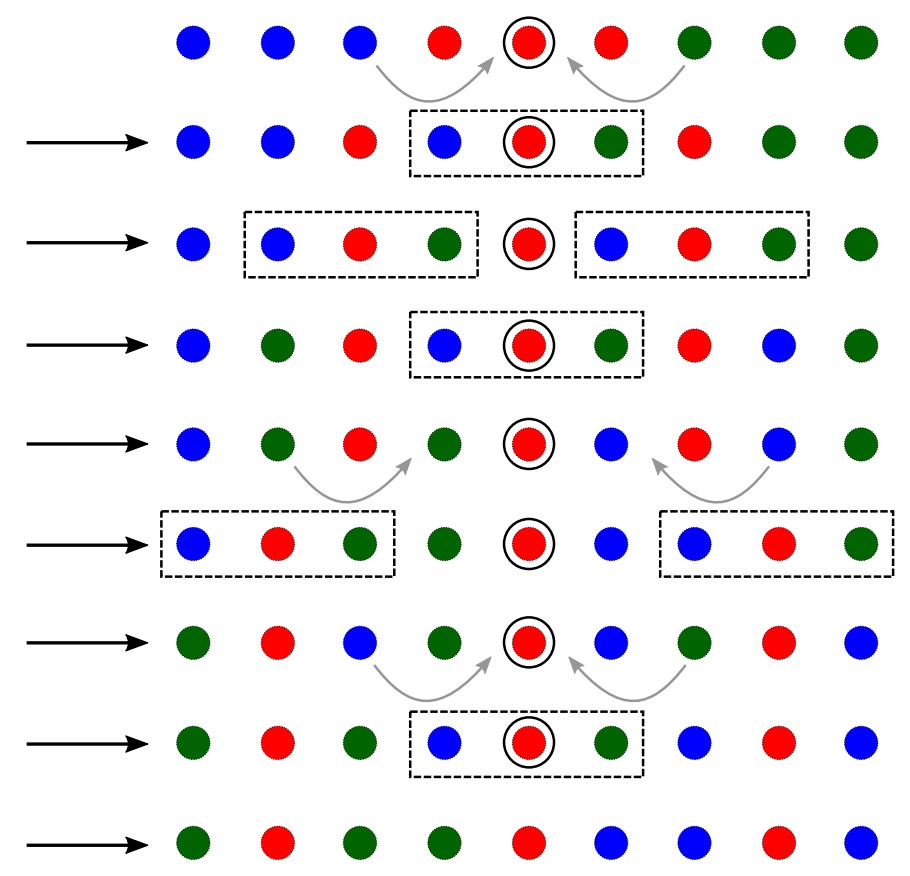

Figure 6. Diagrammatic representation for the scattering processes. The blue, red and green dots stand for particles 1,2 and 3 respectively. The circled red dot denotes the seed particle 2 and the dashed box represent the triple scattering process [123]. We see 7 triple scattering processes in the diagram.

The rest of the scattering cannot involve the triple processes that we are looking for, so we stop here. In the above, the particle in the middle is denoted by italic font 2. This particle is special for our algorithm and is called the seed particle. The triple scattering process which corresponds to the triangle in the previous section is denoted by

$$
\cdots[123] \cdots \longrightarrow \cdots 321 \cdots
$$

The process described in (A.3) can be represented diagrammatically as in figure 6 .

Now we give the general proof. As before, we need to consider several cases while performing the counting. We split the scattering process into two parts. The first part is universal for all the cases. The second part depends on the various cases we consider.

\section{A.1 Universal move}

We start with the following configuration with the seed particle

$$
1^{N_{1}} 2^{N_{2}} 3^{N_{3}}=1^{N_{1}} 2^{S_{2} 2^{N_{2}-S-1}} 3^{N_{3}},
$$

where

$$
S=\left[\frac{N_{1}+N_{2}-N_{3}}{2}\right]
$$


We move the particles $1 / 3$ towards the seed particle from the left/right. The first step is

$$
\begin{aligned}
1^{N_{1}} 2_{22^{N_{2}-S-1} 3^{N_{3}}} & \longrightarrow 1^{N_{1}-1} 2^{S}[123] 2^{N_{2}-S-1} 3^{N_{3}-1} \\
& =1^{N_{1}-1} 2^{S_{3}} 212^{N_{2}-S-1} 3^{N_{3}-1} \\
& =1^{N_{1}-1} 2^{S-1}(23) 2(12) 2^{N_{2}-S-2} 3^{N_{3}-1} .
\end{aligned}
$$

For the $l$-th step, we perform the move

$$
\begin{aligned}
& 1^{N_{1}-l+1} 2^{S-l+1}(23)^{l-1} 2(12)^{l-1} 2^{N_{2}-S-l} 3^{N_{3}-l+1} \\
\longrightarrow & 1^{N_{1}-l} 2^{S-l+1} 1(23)^{l-1} 2(12)^{l-1} 32^{N_{2}-S-l} 3^{N_{3}-l} \\
\longrightarrow & 1^{N_{1}-l} 2^{S-l+1}(32)^{l-1}[123](21)^{l-1} 2^{N_{2}-S-l} 3^{N_{3}-l} \\
\longrightarrow & 1^{N_{1}-l} 2^{S-l+1}(32)^{l-1} 321(21)^{l-1} 2^{N_{2}-S-l} 3^{N_{3}-l} \\
= & 1^{N_{1}-l_{2}} 2^{S-l}(23)^{l} 2(12)^{l_{2}} 2^{N_{2}-S-l-1} 3^{N_{3}-l} .
\end{aligned}
$$

The number of triple scattering processes involved in the $l$-th step is $2 l-1$. We can perform this move until one of the exponents $N_{1}-l, S-1, N_{2}-S-l-1$ and $N_{3}-l$ becomes zero. Therefore we need to distinguish between different cases. First we say without loss of generality $N_{2} \geq N_{3}$ and $N_{2} \geq N_{1}$. We can always obtain this by cyclicity. We consider the case that $N_{1} \leq N_{3}$. The case with $N_{1}>N_{3}$ can be dealt with in a similar way.

\section{A.2 The case $S>N_{1}$ and $N_{3} \geq N_{1}$}

We have $N_{2} \geq N_{1}+N_{3}$. The universal move stops because $N_{1}-l$ becomes zero and we end up with the following configuration:

$$
2^{S-N_{1}}(23)^{N_{1}} 2(12)^{N_{1}} 2^{N_{2}-S-N_{1}-1} 3^{N_{3}-N_{1}}
$$

For the rest of the argument the seed particle is not important anymore, so we treat it as the other 2 particles. We perform the move

$$
\begin{aligned}
& 2^{S-N_{1}}(23)^{N_{1}} 2(12)^{N_{1}} 2^{N_{2}-S-N_{1}-1} 3^{N_{3}-N_{1}} \\
\longrightarrow & 2^{S-N_{1}}(23)^{N_{1}} 2\left[(12)^{N_{1}} 3\right] 2^{N_{2}-S-N_{1}-1} 3^{N_{3}-N_{1}-1} \\
\longrightarrow & 2^{S-N_{1}}(23)^{N_{1}} 23(21)^{N_{1}} 2^{N_{2}-S-N_{1}-1} 3^{N_{3}-N_{1}-1} \\
= & 2^{S-N_{1}}(23)^{N_{1}+1} 2(12)^{N_{1}} 2^{N_{2}-S-N_{1}-2} 3^{N_{3}-N_{1}-1} .
\end{aligned}
$$

This can be done $N_{3}-N_{1}$ times. Each step involves $N_{1}$ triple scattering processes. Finally we end up with the configuration

$$
2^{S-N_{1}}(23)^{N_{3}} 2(12)^{N_{1}} 2^{N_{2}-S-N_{3}-1} .
$$

In this two-part procedure, we count the total number of triangle scattering processes

$$
T\left(N_{1}, N_{2}, N_{3}\right)=\sum_{l=1}^{N_{1}}(2 l-1)+N_{1}\left(N_{3}-N_{1}\right)=N_{1} N_{3},
$$

which matches what we had found in section 3 for cases I and III. 


\section{A.3 The case $S \leq N_{1}$ and $N_{3}>N_{1}$}

Here the universal move stops because $S-l$ becomes zero. We end up with

$$
1^{N_{1}-S}(23)^{S_{2}}(12)^{S_{2}}{ }^{N_{2}-2 S-1} 3^{N_{3}-S} .
$$

To obtain further triple scattering processes, we first perform the move

$$
\begin{aligned}
& 1^{N_{1}-S}(23)^{S} 2(12)^{S_{2}} 2^{N_{2}-2 S-1} 3^{N_{3}-S} \\
= & 1^{N_{1}-S-1}\left[1(23)^{S}\right] 2(12)^{S} 2^{N_{2}-2 S-1} 3^{N_{3}-S} \\
\longrightarrow & 1^{N_{1}-S-1}(32)^{S} 12(12)^{S} 2^{N_{2}-2 S-1} 3^{N_{3}-S} \\
= & 1^{N_{1}-S-1} 3(23)^{S-1} 2(12)^{S+1} 2^{N_{2}-2 S-1} 3^{N_{3}-S} .
\end{aligned}
$$

This move involves $S$ triple scattering processes. Then we perform the following recursively: at step $l$, we have

$$
\begin{aligned}
& 1^{N_{1}-S-l} 3^{l}(23)^{S-1} 2(12)^{S+l} 2^{N_{2}-2 S-l} 3^{N_{3}-S-l+1} \\
\longrightarrow & 1^{N_{1}-S-l-1} 3^{l}\left[1(23)^{S-1}\right] 2\left[(12)^{S+l} 3\right] 2^{N_{2}-2 S-l} 3^{N_{3}-S-l} \\
\longrightarrow & 1^{N_{1}-S-l-1} 3^{l}(32)^{S-1}[123](12)^{S+l} 2^{N_{2}-2 S-l} 3^{N_{3}-S-l} \\
\longrightarrow & 1^{N_{1}-S-l-1} 3^{l}(32)^{S-1} 321(21)^{S+l} 2^{N_{2}-2 S-l} 3^{N_{3}-S-l} \\
= & 1^{N_{1}-S-l-1} 3^{l+1}(23)^{S-1} 2(12)^{S+l+1} 2^{N_{2}-2 S-l-1} 3^{N_{3}-S-l} .
\end{aligned}
$$

When the process ends depends on whether $N_{1}-S-1$ or $N_{2}-2 S-1$ is larger. We separately consider these cases.

1. First, let $N_{2}-N_{1} \geq S$ so that the process ends because $N_{1}-S-l-1$ becomes zero. We end on

$$
3^{N_{1}-S}(23)^{S-1} 2(12)^{N_{1}} 2^{N_{2}-N_{1}-S_{3}}{ }^{N_{3}-N_{1}+1} .
$$

To proceed, we perform the following recursive move: for step $l$, we have

$$
\begin{aligned}
& 3^{N_{1}-S}(23)^{S+l-2} 2(12)^{N_{1}} 2^{N_{2}-N_{1}-S-l+1} 3^{N_{3}-N_{1}-l+2} \\
\longrightarrow & 3^{N_{1}-S}(23)^{S+l-2} 2\left[(12)^{N_{1}} 3\right] 2^{N_{2}-N_{1}-S-l+1} 3^{N_{3}-N_{1}-l+1} \\
\longrightarrow & 3^{N_{1}-S}(23)^{S+l-2} 23(21)^{N_{1}} 2^{N_{2}-N_{1}-S-l+1} 3^{N_{3}-N_{1}-l+1} \\
= & 3^{N_{1}-S}(23)^{S+l-1} 2(12)^{N_{1}} 2^{N_{2}-N_{1}-S-l} 3^{N_{3}-N_{1}-l+1} .
\end{aligned}
$$

Each step involves $N_{1}$ and will stop for $l=N_{2}-N_{1}-S$. So the total number of triple scattering processes is $N_{1}\left(N_{2}-N_{1}-S\right)$. We end up with the following configuration

$$
3^{N_{1}-S}(23)^{N_{2}-N_{1}-1} 2(12)^{N_{1}} 3^{N_{3}-N_{2}+S+1} .
$$

As the last step, we perform the following recursive move, for the $l$-th step

$$
\begin{aligned}
& 3^{N_{1}-S}(23)^{N_{2}-N_{1}+l-1} 2(12)^{N_{1}-l} 1^{l} 3^{N_{3}-N_{2}+S-l+1} \\
\longrightarrow & 3^{N_{1}-S}(23)^{N_{2}-N_{1}+l-1} 2\left[(12)^{N_{1}-l} 3\right] 11^{l_{3}} 3^{N_{3}-N_{2}+S-l} \\
\longrightarrow & 3^{N_{1}-S}(23)^{N_{2}-N_{1}+l-1} 23(21)^{N_{1}-l} 1^{l} 3^{N_{3}-N_{2}+S-l} \\
= & 3^{N_{1}-S}(23)^{N_{2}-N_{1}+l} 2(12)^{N_{1}-l-1} 1^{l+1} 3^{N_{3}-N_{2}+S-l} .
\end{aligned}
$$


This stops at $l=N_{3}-N_{2}+S$ and end up with the following configuration

$$
3^{N_{1}-S}(23)^{N_{3}-N_{1}+S_{2}} 2(12)^{N_{1}+N_{2}-N_{3}-S-1} 1^{N_{3}-N_{2}+S+1} .
$$

We can not produce further triple scattering processes. Summing up the number of triple scattering processes, we obtain

$$
\begin{aligned}
T\left(N_{1}, N_{2}, N_{3}\right)= & \sum_{l=1}^{S}(2 l-1)+S+\sum_{l=1}^{N_{1}-S-1}(2 S+l)+N_{1}\left(N_{2}-N_{1}-S\right) \\
& +N_{1}+\sum_{l=1}^{N_{3}-N_{2}+S}\left(N_{1}-l\right) \\
= & -\frac{1}{4}\left(N_{1}^{2}+N_{2}^{2}+N_{3}^{2}-2 N_{1} N_{2}-2 N_{1} N_{3}-2 N_{2} N_{3}-c\right),
\end{aligned}
$$

where $c=\bmod \left[N_{1}+N_{2}+N_{3}, 2\right]$. Using the result, we find that the order $g^{p}$ is given by

$$
p=\sum_{i=1}^{3} \ell_{i} N_{i}+\frac{1+(-1)^{\sum_{i} N_{i}}}{2} .
$$

2. The case $N_{2}-N_{1}<S$, the process ends because $N_{2}-2 S-1$ becomes zero. We end up with the following configuration

$$
1^{N_{1}-N_{2}+S_{3}}{ }^{N_{2}-2 S}(23)^{S-1} 2(12)^{N_{2}-S_{3}}{ }^{N_{3}-N_{2}+S+1} .
$$

We now perform the following recursive move at $l$-th step. Here we get $N_{2}-S-l+1$ triples.

$$
\begin{aligned}
& 1^{N_{1}-N_{2}+S_{3}} 3^{N_{2}-2 S}(23)^{S+l-2} 2(12)^{N_{2}-S-l+1} 1^{l-1} 3^{N_{3}-N_{2}+S+2-l} \\
\longrightarrow & 1^{N_{1}-N_{2}+S} 3^{N_{2}-2 S}(23)^{S+l-1} 2(12)^{N_{2}-S-l} 1^{l} 3^{N_{3}-N_{2}+S+1-l} .
\end{aligned}
$$

This procedure stops when $N_{3}-N_{2}+S+1-l$ goes to zero. We get the following configuration:

$$
1^{N_{1}-N_{2}+S_{3}} 3^{N_{2}-2 S}(23)^{N_{3}-N_{2}+2 S} 2(12)^{2 N_{2}-2 S-N_{3}-1} 1^{N_{3}-N_{2}+S+1} .
$$

We now move all the 1 from the left across all (23). At $l$-th step again we get the following move:

$$
\begin{aligned}
& 1^{N_{1}-N_{2}+S-l+1} 3^{N_{2}-2 S+l-1}(23)^{N_{3}-N_{2}+2 S-l+1} 2(12)^{2 N_{2}-2 S-N_{3}-l} 1^{N_{3}-N_{2}+S+1} \\
\longrightarrow & 1^{N_{1}-N_{2}+S-l} 3^{N_{2}-2 S+l}(23)^{N_{3}-N_{2}+2 S-l} 2(12)^{2 N_{2}-2 S-N_{3}-l-1} 1^{N_{3}-N_{2}+S+1} .
\end{aligned}
$$

By doing this step we get $N_{3}-N_{2}+2 S-l+1$ new triples. This is possible until $N_{1}-N_{2}+S-l$ goes to zero. Then we end up with:

$$
3^{N_{1}-S}(23)^{N_{3}-N_{1}+S_{2}} 2(12)^{N_{2}-S-N_{3}+N_{1}-1} 1^{N_{3}-N_{2}+S+1} .
$$


Here we cannot produce any more triples again. Counting all triples we get the following.

$$
\begin{aligned}
T\left(N_{1}, N_{2}, N_{3}\right)= & \sum_{l=1}^{S}(2 l-1)+S+\sum_{l=1}^{N_{2}-2 S-1}(2 S+l)+\sum_{l=1}^{N_{3}-N_{2}+S+1}\left(N_{2}-S-l+1\right) \\
& +\sum_{l=1}^{N_{1}-N_{2}+S}\left(N_{3}-N_{2}+2 S-l+1\right)
\end{aligned}
$$

Simplifying this expression we obtain a result for $p$ similar to (A.22).

\section{A.4 The case $N_{3}=N_{1}$ and $S \leq N_{3}$}

In this case, we have again $N_{2} \leq N_{1}+N_{3}=2 N_{3}$. With this constraints we get $S=\left[\frac{N_{2}}{2}\right]$. The universal move stops because $N_{2}-S-1-l$ becomes zero. We now consider to different cases.

1. We consider first that $N_{2}$ is even, then $S=\frac{N_{2}}{2}$. So we end up with the following configuration:

$$
1^{N_{3}-S+1} 2(23)^{S-1} 2(12)^{S-1} 3^{N_{3}-S+1} .
$$

We now perform the following move and get $S-1$ triples.

$$
\begin{aligned}
& 1^{N_{3}-S+1} 2(23)^{S-1} 2(12)^{S-1} 3^{N_{3}-S+1} \\
\longrightarrow & 1^{N_{3}-S}(23)^{S-1} 2(12)^{S} 3^{N_{3}-S+1} .
\end{aligned}
$$

Now we move recursively a 1 from the left and a 3 from the right simultaneously to the middle as shown in (A.31). Here we get at the $l$-th step $2 S$ new triples.

$$
\begin{aligned}
& 1^{N_{3}-S-l+1} 3^{l-1}(23)^{S-1} 2(12)^{S} 1^{l-1} 3^{N_{3}-S+2-l} \\
= & 1^{N_{3}-S-l} 3^{l-1}\left[1(23)^{S-1}\right] 2\left[(12)^{S} 3\right] 1^{l-1} 3^{N_{3}-S+1-l} \\
\longrightarrow & 1^{N_{3}-S-l} 3^{l-1}(32)^{S-1}[123](21)^{S} 1^{l-1} 3^{N_{3}-S+1-l} \\
\longrightarrow & 1^{N_{3}-S-l} 3^{l}(23)^{S-1} 2(12)^{S} 1^{l} 3^{N_{3}-S+1-l} .
\end{aligned}
$$

This recursion ends if $N 3-S-l$ becomes zero and we end up with the following:

$$
3^{N_{3}-S}(23)^{S-1} 2(12)^{S} 1^{N 3-S} 3 .
$$

By moving the 3 on the right through the (12)'s we get $S$ triples and cannot perform other triple moves then. All in all the sum of the triples is

$$
\begin{aligned}
T\left(N_{3}, N_{2}, N_{3}\right) & =\sum_{l=1}^{S-1}+S-1+\sum_{l=1}^{N_{3}-S}(2 S)+S \\
& =-\frac{N_{2}^{2}}{4}+N_{2} N_{3} .
\end{aligned}
$$

This is the same like in (A.21) with $N_{1}=N_{3}$ and $N_{2}$ even. So we end up with formula (A.22) for $p$. 
2. Now we consider that $N_{2}$ is odd, then $S=\frac{N 2}{2}-\frac{1}{2}$. From universal move we end up with:

$$
1^{N_{3}-S_{2}}(23)^{S_{2}}(12)^{S_{3}}{ }^{N_{3}-S}
$$

Now we move recursively a 1 from the left and a 3 from the right to the middle as shown below. At $l$-th step we get $2 S+1$ triples.

$$
\begin{aligned}
& 1^{N_{3}-S-l+1} 3^{l-1}(23)^{S} 2(12)^{S} 1^{l-1} 3^{N_{3}-S-l+1} \\
= & 1^{N_{3}-S-l} 3^{l-1}\left[1(23)^{S}\right] 2\left[(12)^{S} 3\right] 1^{l-1} 3^{N_{3}-S-l} \\
\longrightarrow & 1^{N_{3}-S-l} 3^{l-1}(32)^{S}[123](21)^{S} 1^{l-1} 3^{N_{3}-S-l} \\
\longrightarrow & 1^{N_{3}-S-l} 3^{l}(23)^{S} 2(12)^{S} 1^{l} 3^{N_{3}-S-l} .
\end{aligned}
$$

This procedure ends if $N_{3}-S-l$ becomes zero. After that it is not possible to do another triple move. All in all the sum of the triples is

$$
\begin{aligned}
T\left(N_{1}, N_{2}, N_{3}\right) & =\sum_{l=1}^{S}(2 l-1)+\sum_{l=1}^{N 3-S}(2 S+1) \\
& =-\frac{1}{4}\left(N_{2}^{2}-4 N_{2} N_{3}-1\right) .
\end{aligned}
$$

Which is also the same like in (A.21) with $N_{1}=N_{3}$ and $N_{2}$ odd. So we end up with formula (A.22) for $p$.

\section{B General configurations involving physical magnons}

In the appendix we extend the discussion of section 3.4 by considering three families of physical magnons $\mathbf{x}, \mathbf{y}, \mathbf{z}$ as well as three families of mirror magnons $\mathbf{u}, \mathbf{v}, \mathbf{w}$, see eq. (2.1), filling all edges of a hexagon. We label the number of mirror excitations by $N_{1}, N_{2}, N_{3}$ as above, while the number of physical ones is $M_{1}, M_{2}, M_{3}$. We take the convention that there are $N_{i}$ mirror magnons on the edge opposite to the one with $M_{i}$ physical magnons. As discussed above, see eq. (2.12), the worst-case scenario for our estimate occurs when all physical magnons are so(6) excitations. In that case, scattering mirror-physical magnons from opposite edges yields a power of $g^{-1}$, so that we naïvely expect the hexagon to acquire an order $g^{p}$, where the contribution of physical magnons shifts the results of section 3.3 as

$$
p \rightarrow p-\sum_{i=1}^{3} M_{i} N_{i}, \quad \text { naïvely. }
$$

However, as discussed in the main text, certain multiple scattering events scale better than predicted by (2.12). Looking at eq. (2.15) and recalling the discussion of section 3.4 we see that these configurations are cone-like sequences of mirror-physical-mirror scattering events. 

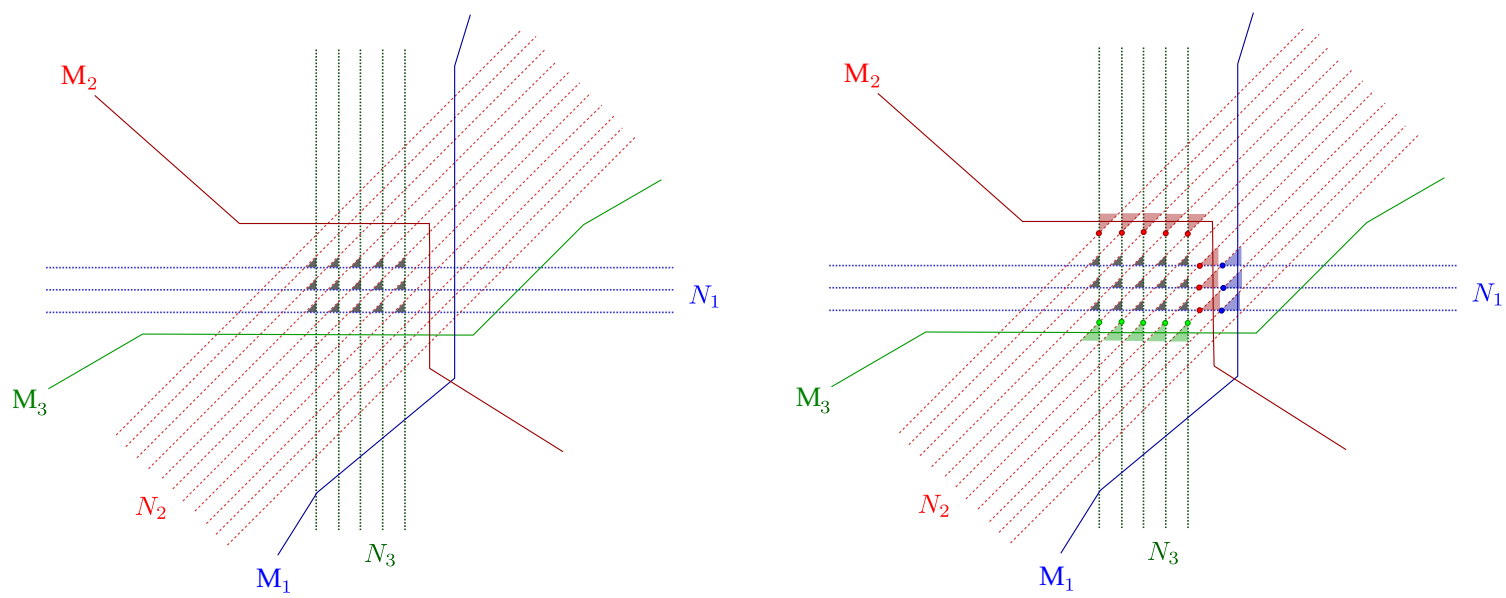

Figure 7. Scattering of physical and mirror magnons in "case III". A strand of $M_{i}$ physical magnons is indicated by a single thick solid line, while individual mirror magnons are indicated by dashed lines. There are $M_{i}$ physical magnons sitting on the edge opposite to the mirror one containing $N_{i}$ virtual magnons. Left panel: we count how many times physical magnons scatter with mirror ones from the opposite edge. Clearly this happens $N_{i} M_{i}$ times for $i=1, \ldots 3$. Right panel: we identify the "cones" as in eq. (2.15). In the drawing for the present case there are $N_{1}$ and $N_{3}$ such cones for the "short" edges, while for the "longer" one (here $N_{2}>N_{1}+N_{3}+1$ ) there are $\left(N_{1}+N_{3}\right)$ cones.

Estimate of cones, case III and I. Let us estimate how many such "cones" can appear when computing the hexagon form factor. It is convenient to work diagrammatically, distinguishing the cases of the main text. We start from "case III" where $N_{2}>N_{1}+N_{3}+1$ as discussed in section 3.4, but here we consider an arbitrary numbers of physical magnons $M_{1}, M_{2}$ and $M_{3}$, see figure 7 . We see that for the "short" edges $N_{1}, N_{3}$ we have $N_{1}$ and $N_{3}$ cones, while for the longer on we have $N_{1}+N_{3}$ cones. As each cone contains $M_{i}$ physical lines we get the improved estimate

$$
p \rightarrow p-M_{2}\left(N_{2}-N_{1}-N_{3}\right)
$$

This happens to be the very same as the estimate for the simpler setup of section 3.4, namely

$$
p=\sum_{i=1}^{3} N_{i} \ell_{i}+\left(N_{2}-N_{1}-N_{3}\right)\left(N_{2}-N_{1}-N_{3}-M_{2}\right) .
$$

Therefore, the estimate is bounded from below as argued in the main text. Notice that the other similar cases, and in particular case I, can be obtained by relabelling the indices $i=1,2,3$, keeping track of which $N_{i}$ is larger than the sum of the other two.

In figure 6 we could equally have passed the solid line representing the scattering of the $M_{1}$ physical excitations to the left of the central region of the panels. Then equation (2.15) directly yields the aforementioned estimate because none of the cones is passed by two strands of physical magnons. The pictures in figure 6 remain correct even if the $M_{1}$ and $M_{2}$ physical magnons pass through cones with the same "root" thanks to equation (2.16). 

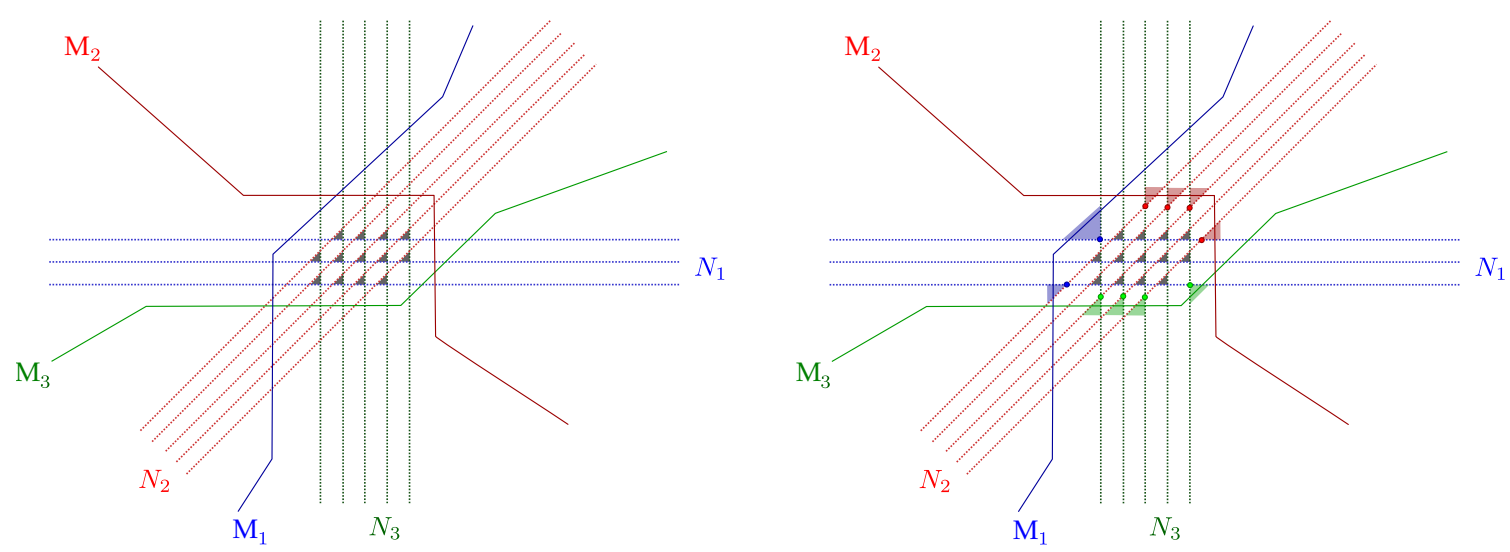

Figure 8. Scattering of physical and mirror magnons in "case II". A strand of $M_{i}$ physical magnons is indicated by a single thick solid line, while individual mirror magnons are indicated by dashed lines (the lines are arranged differently from figure 7 for clarity). There are $M_{i}$ physical magnons sitting on the edge opposite to the mirror one containing $N_{i}$ mirror magnons. Left panel: we count how many times physical magnons scatter with mirror ones from the opposite edge, which happens $N_{i} M_{i}$ times for $i=1, \ldots 3$. Right panel: we identify the "cones" as in eq. (2.15). In the drawing for this case there are $\left(N_{i}-1\right)$ cones for each type of lines.

Estimate of cones, case II. In this case all numbers of mirror particles $N_{i}$ are of the same order. More specifically, no $N_{i}$ is bigger than the sum of the other two. Following figure 8 we see that there are $\left(N_{i}-1\right)$ cones for any $i=1, \ldots 3$, giving an improvement of $M_{i}\left(N_{i}-1\right)$. As a result, we find

$$
p \rightarrow p-\sum_{i=1}^{3} M_{i},
$$

which is just an $N_{i}$-independent shift. Hence the final result for $p$ is in this case

$$
p=\sum_{i=1}^{3}\left(N_{i} \ell_{i}-M_{i}\right)+\frac{1+(-1)^{\sum_{i} N_{i}}}{2} .
$$

Open Access. This article is distributed under the terms of the Creative Commons Attribution License (CC-BY 4.0), which permits any use, distribution and reproduction in any medium, provided the original author(s) and source are credited.

\section{References}

[1] J.M. Maldacena, The large $N$ limit of superconformal field theories and supergravity, Int. $J$. Theor. Phys. 38 (1999) 1113 [hep-th/9711200] [INSPIRE].

[2] E. Witten, Anti-de Sitter space and holography, Adv. Theor. Math. Phys. 2 (1998) 253 [hep-th/9802150] [INSPIRE].

[3] S.S. Gubser, I.R. Klebanov and A.M. Polyakov, Gauge theory correlators from noncritical string theory, Phys. Lett. B 428 (1998) 105 [hep-th/9802109] [INSPIRE]. 
[4] G. 't Hooft, A Planar Diagram Theory for Strong Interactions, Nucl. Phys. B 72 (1974) 461 [INSPIRE].

[5] J.A. Minahan and K. Zarembo, The Bethe ansatz for $\mathcal{N}=4$ superYang-Mills, JHEP 03 (2003) 013 [hep-th/0212208] [INSPIRE].

[6] G. Arutyunov and S. Frolov, Foundations of the AdS $S_{5} \times S^{5}$ Superstring. Part I, J. Phys. A 42 (2009) 254003 [arXiv:0901.4937] [inSPIRE].

[7] N. Beisert et al., Review of AdS/CFT Integrability: An Overview, Lett. Math. Phys. 99 (2012) 3 [arXiv: 1012.3982] [INSPIRE].

[8] B. Basso, S. Komatsu and P. Vieira, Structure Constants and Integrable Bootstrap in Planar $N=4 S Y M$ Theory, arXiv:1505.06745 [INSPIRE].

[9] B. Eden and A. Sfondrini, Tessellating cushions: four-point functions in $\mathcal{N}=4$ SYM, JHEP 10 (2017) 098 [arXiv:1611.05436] [INSPIRE].

[10] T. Fleury and S. Komatsu, Hexagonalization of Correlation Functions, JHEP 01 (2017) 130 [arXiv: 1611.05577] [INSPIRE].

[11] B. Eden, Y. Jiang, D. le Plat and A. Sfondrini, Colour-dressed hexagon tessellations for correlation functions and non-planar corrections, JHEP 02 (2018) 170 [arXiv:1710.10212] [INSPIRE].

[12] T. Bargheer, J. Caetano, T. Fleury, S. Komatsu and P. Vieira, Handling Handles I: Nonplanar Integrability, arXiv:1711.05326 [INSPIRE].

[13] W. Carlson, R. de Mello Koch and H. Lin, Nonplanar Integrability, JHEP 03 (2011) 105 [arXiv: 1101.5404] [INSPIRE].

[14] R. de Mello Koch, M. Kim and H.J.R. Zyl, Integrable Subsectors from Holography, JHEP 05 (2018) 198 [arXiv: 1802.01367] [INSPIRE].

[15] C. Kristjansen, Review of AdS/CFT Integrability, Chapter IV.1: Aspects of Non-Planarity, Lett. Math. Phys. 99 (2012) 349 [arXiv:1012.3997] [InSPIRE].

[16] N. Beisert and M. Staudacher, Long-range PSU $(2,2 \mid 4)$ Bethe Ansatze for gauge theory and strings, Nucl. Phys. B 727 (2005) 1 [hep-th/0504190] [INSPIRE].

[17] J. Ambjørn, R.A. Janik and C. Kristjansen, Wrapping interactions and a new source of corrections to the spin-chain/string duality, Nucl. Phys. B 736 (2006) 288 [hep-th/0510171] [INSPIRE].

[18] M. Lüscher, Volume Dependence of the Energy Spectrum in Massive Quantum Field Theories. 1. Stable Particle States, Commun. Math. Phys. 104 (1986) 177 [InSPIRE].

[19] M. Lüscher, Volume Dependence of the Energy Spectrum in Massive Quantum Field Theories. 2. Scattering States, Commun. Math. Phys. 105 (1986) 153 [InSPIRE].

[20] B. Eden and A. Sfondrini, Three-point functions in $\mathcal{N}=4 S Y M$ : the hexagon proposal at three loops, JHEP 02 (2016) 165 [arXiv: 1510.01242] [INSPIRE].

[21] B. Basso, V. Goncalves, S. Komatsu and P. Vieira, Gluing Hexagons at Three Loops, Nucl. Phys. B 907 (2016) 695 [arXiv:1510.01683] [InSPIRE].

[22] B. Basso, V. Goncalves and S. Komatsu, Structure constants at wrapping order, JHEP 05 (2017) 124 [arXiv:1702.02154] [INSPIRE]. 
[23] T. Fleury and S. Komatsu, Hexagonalization of Correlation Functions II: Two-Particle Contributions, JHEP 02 (2018) 177 [arXiv:1711.05327] [INSPIRE].

[24] N. Beisert, The SU(2|2) dynamic S-matrix, Adv. Theor. Math. Phys. 12 (2008) 945 [hep-th/0511082] [INSPIRE].

[25] G. Arutyunov, M. de Leeuw and A. Torrielli, The Bound State S-matrix for Ad $S_{5} \times S^{5}$ Superstring, Nucl. Phys. B 819 (2009) 319 [arXiv:0902.0183] [INSPIRE].

[26] N. Beisert, B. Eden and M. Staudacher, Transcendentality and Crossing, J. Stat. Mech. 0701 (2007) P01021 [hep-th/0610251] [INSPIRE].

[27] G. Arutyunov, S. Frolov and M. Zamaklar, The Zamolodchikov-Faddeev algebra for $A d S_{5} \times S^{5}$ superstring, JHEP 04 (2007) 002 [hep-th/0612229] [INSPIRE].

[28] G. Arutyunov and S. Frolov, The Dressing Factor and Crossing Equations, J. Phys. A 42 (2009) 425401 [arXiv:0904.4575] [INSPIRE].

[29] J.M. Drummond, J. Henn, V.A. Smirnov and E. Sokatchev, Magic identities for conformal four-point integrals, JHEP 01 (2007) 064 [hep-th/0607160] [INSPIRE].

[30] E. D'Hoker, D.Z. Freedman, S.D. Mathur, A. Matusis and L. Rastelli, Extremal correlators in the AdS/CFT correspondence, hep-th/9908160 [INSPIRE].

[31] M. Bianchi and S. Kovacs, Nonrenormalization of extremal correlators in N $=4 S Y M$ theory, Phys. Lett. B 468 (1999) 102 [hep-th/9910016] [INSPIRE].

[32] B. Eden, P.S. Howe, C. Schubert, E. Sokatchev and P.C. West, Extremal correlators in four-dimensional SCFT, Phys. Lett. B 472 (2000) 323 [hep-th/9910150] [INSPIRE].

[33] D. Chicherin, J. Drummond, P. Heslop and E. Sokatchev, All three-loop four-point correlators of half-BPS operators in planar $\mathcal{N}=4$ SYM, JHEP 08 (2016) 053 [arXiv: 1512.02926] [INSPIRE].

[34] A. Sfondrini, Towards integrability for AdS3/CFT 2 , J. Phys. A 48 (2015) 023001 [arXiv: 1406.2971] [INSPIRE].

[35] M. Baggio and A. Sfondrini, Strings on NS-NS Backgrounds as Integrable Deformations, Phys. Rev. D 98 (2018) 021902 [arXiv: 1804.01998] [INSPIRE].

[36] A. Dei and A. Sfondrini, Integrable spin chain for stringy Wess-Zumino-Witten models, JHEP 07 (2018) 109 [arXiv: 1806.00422] [INSPIRE]. 